\title{
Supersymmetry versus Integrability in two-dimensional Classical Mechanics
}

\author{
A. Alonso Izquierdo ${ }^{(a, b)}$, M.A. González León ${ }^{(b)}$, \\ J. Mateos Guilarte ${ }^{(c)}$, and M. de la Torre Mayado ${ }^{(c)}$ \\ (a) DAMPT, Cambridge University, U.K. \\ (b) Departamento de Matemática Aplicada, Universidad de Salamanca, SPAIN \\ (c) Departamento de Física, Universidad de Salamanca, SPAIN
}

\begin{abstract}
Supersymmetric extensions of Hamilton-Jacobi separable Liouville mechanical systems with two degrees of freedom are defined. It is shown that supersymmetry can be implemented in this type of systems in two independent ways. The structure of the constants of motion is unveiled and the entanglement between integrability and supersymmetry is explored.
\end{abstract}

\section{Introduction}

Since the discovery of superstrings [1] and non-linear supersymmetric field theoretical models [2] at the beginning of the seventies in the past century supersymmetry has become an extremely active area of research in both theoretical physics and mathematics. In particular, supersymmetric non-Abelian gauge theories in several dimensions are of broad interest passing through the phenomenology of elementary particles [3] to differential invariants of four manifolds [4].

At a very early stage in the development of this matter, several researchers started to focus on understanding Bose/Fermi symmetry in the realms of classical and quantum mechanics of finite dimensional dynamical systems [5], [6]. In supersymmetric quantum mechanics, the fermionic variables are realized as the generators of a Clifford algebra satisfying the quantization rules: $\left\{\theta^{j}(t), \theta^{k}(t)\right\}=i \hbar \delta^{j k}$. Therefore, they are (Grassmann) anticommuting variables $\left\{\theta^{j}, \theta^{k}\right\}=0$ at the $\hbar=0$ classical limit [7]. The geometry of manifolds including variables of this kind is described in DeWitt's book [8]. Thus, in classical supersymmetric theories the configuration space is a supermanifold, in DeWitt's sense: the dynamical variables take their values in a Grassman algebra $\mathcal{B}_{L}$. Any element of $\mathcal{B}_{L}$ is the combination of the $L$ generators $\xi_{A_{k}}^{i_{r}}, b=b_{0} \mathbf{1}+\sum b_{i_{1} \cdots i_{n}}^{A_{1} \cdots A_{m}} \xi_{A_{1}}^{i_{1}} \cdots \xi_{A_{m}}^{i_{n}}$, where the coefficients $b_{i_{1} \cdots i_{n}}^{A_{1} \cdots A_{m}}$ are real numbers. $b_{0}$ is usually referred to as the body of the supernumber $b$ whereas the sum of the other terms in the Grassman expansion is accordingly named as the soul of $b$. The Lagrangian formalism of classical mechanics can be extended to the supersymmetric framework and the Hamiltonian formalism can also be implemented in supersymmetric 
dynamical systems, see $[9,10]$. Several simple mechanical models, with bosonic and fermionic dynamical variables valued in a Grassman algebra have been investigated by Casalbuoni [5], Berezin and Marinov [6], Junker and Matthiesen [11]. Manton and Heumann have recently improved on these works, obtaining supersolutions in several interesting models $[12,13,14]$

The main theme in the present work is to investigate the interplay between supersymmetry and integrability in dynamical systems with two bosonic degrees of freedom. There is a broad class of two-dimensional classical systems, called Liouville systems, that, besides being completely integrable, have the stronger property of being Hamilton-Jacobi-separable, see [15]. Amongst them rank some important physical systems: the two-dimensional Kepler and two Newtonian centers of force problems, the Garnier system [16], to quote just three. The existence of a second first-integral in involution with the Hamiltonian guarantees, via Liouville's theorem, complete integrability. The second invariant is usually referred as corresponding to hidden symmetries; we shall show that, via the introduction of generalized momenta, these invariants can be related with well known constants of motion. Moreover, the hallmark of Liouville's systems, the separability of the Hamilton-Jacobi equation, reduces the analytical solution to independent quadratures in the two degrees of freedom.

In our attempt to build supersymmetry in systems of the Liouville type we face three main tasks:

1. Construction of supersymmetric extensions to be called superLiouville models.

2. The search for the second invariant in the supersymmetric framework.

3. To look at the fate of Hamilton-Jacobi separability when fermionic degrees of freedom are added in a supersymmetric way

There are precedents of the study of constant of motions in supersymmetric classical mechanics in the literature. Plyushchay identified invariants in supersymmetric classical and pseudo classical mechanical models involving one bosonic degree of freedom in [17]. Heumann [14] and Wipf et al [18] dealt with the supersymmetric version of the Runge-Lenz vector respectively in the classical and quantum supersymmetric Coulomb problems. Whether or not the invariants of a classical system promote invariants in the supersymmetric extension is a crucial question regarding integrability. There are also precedents of connecting classical integrable systems with supersymmetry, see the recent paper [19] where the classical limit of SUSY quantum mechanics is used to define two dimensional integrable systems. Connections between non-linear supersymmetry and quasiexactly solvable systems have been pointed out in the interesting papers [20] and [21], where the dynamics of a charged spin $\frac{1}{2}$ in a magnetic field is described using ideas of SUSY quantum mechanics.

In supersymmetric models with one bosonic degree of freedom the Hamiltonian and the supercharges are obvious (non-independent) first-integrals. The analytic solution of these models can thus be reduced to quadratures via the Grassman/Manton/Heumann expansion as in [13]. There also exist some combinations between the fermionic variables which are conserved, such as was shown in [17]. The situation is more difficult in models with two bosonic degrees of freedom, where the identification of invariants is an arduous task even within the purely bosonic framework. Regarding this latter point, one of most celebrated work is that of Hietarinta [22]: all the 
integrable systems of type $H=\frac{1}{2} p_{x_{1}}^{2}+\frac{1}{2} p_{x_{2}}^{2}+U\left(x_{1}, x_{2}\right)$ are analyzed, with $U$ a polynomial in $x_{1}$ and $x_{2}$ of degree 5 or less, and the second invariant is at most of fourth order in $p_{x}$ and $p_{y}$. The procedure used is conceptually simple: the existence of a second invariant $I_{2}$ in involution with the Hamiltonian guarantees classical integrability in two-dimensional systems. A polynomial of any order in the coordinates, but at most of degree 4 in the momenta with arbitrary coefficients, is proposed as a candidate to become the second invariant $I$. Accordingly, the Poisson bracket $\{H, I\}_{P}$ is computed. Systems where $I$ can be found such that $\{H, I\}_{P}=0$ are integrable. This strategy has been followed in other works $[23,24]$ and was extended to supersymmetric systems in [25]. Here, we shall apply again Hietarinta's method to two-dimensional supersymmetric classical mechanics in the search for the second invariant in superLiouville models. This will be possible because the Hamiltonian formalism has also been well-defined in the supersymmetric framework [10].

Below we offer the conclusions that we have reached with respect to the three tasks:

1. There exist $\mathcal{N}=2$ extended supersymetric versions of Liouville models. Usually, interactions in supersymmetric theories are determined from the superpotential. The surprise is that in superLiouville models one can choose between two different superpotentials leading to different supersymmetric dynamics. The reason is that the Hamilton-Jacobi equation theory of the parent Liouville model admits four different solutions for the Hamilton characteristic function ( the superpotential ). Two of the solutions differ from the other two by a global sign that makes no difference at all. The other two induce different Bose/Fermi Yukawa couplings.

2. There exist second invariants in other the superLiouville models. The Bose contribution to the Hamiltonian does not depend on the choice of the superpotential but the Fermi contribution, the Yukawa couplings, is different for the non-equivalent superpotentials. Exactly the same situation occurs with respect to the second invariants.

3. The superLiouville models are not Hamilton-Jacobi separable. The Yukawa terms spoil the separability of the two degrees of freedom.

On the physical side, we mention two applications of supersymmetric classical systems. First, the structure of the fermionic contribution to the second invariant naturally shows how the spin of the superparticle is determined as a quadratic product of Grassman variables: $s^{12}=i \theta_{\alpha}^{1} \theta_{\alpha}^{2}$. This explanation for the fermionic degrees of freedom comes back to [6] and was given a deep group theoretical meaning by Azcarraga and Lukierski in Reference [26]. Second, $\mathcal{N}=2$ superLiouville models can be understood as the dimensional reduction of $\mathcal{N}=1$ supersymmetric field theory in $(1+1)$-dimensions. From this point of view the separatrix ( finite action ) supertrajectories are seen as the BPS superkinks of the field theory ( supersymmetric BPS domain walls in $(3+1)$ dimensions). In References [27, 28, 29] we have approached the problem from this angle of attack.

The organization of the paper is as follows: In Section $\S 2$ we describe $\mathcal{N}=2$ supersymmetric classical mechanics, both in Euclidean and Riemannian 2D manifolds; the notations and conventions are introduced also in this Section. Section $\S 3$ is devoted to defining $\mathcal{N}=2$ superLiouville systems after a rapid summary of the properties of the parent Liouville models. In Section $\S 4$, 
the second ( and other ) invariants of the superLiouville models are identified, following the above mentioned Hietarinta strategy. Finally, in Section $\S 5$ a procedure is outlined to generalize the Hamilton-Jacobi method to the supersymmetric framework. It is shown how to search for the supersolutions of the system in a layer-by-layer resolution.

\section{$2 \mathcal{N}=2$ supersymmetric classical mechanics}

In this Section we introduce $\mathcal{N}=2$ super-symmetric classical mechanics, which can also be described as a dimensional reduction of $\mathcal{N}=1$ supersymmetric $(1+1)$-dimensional field theory. We restrict ourselves to models with two bosonic degrees of freedom.

There is in the whole formalism an underlying Grassman algebra $\mathcal{B}_{L}$ with $L$ odd generators $\xi_{A}$ such that $\xi_{A} \xi_{B}=-\xi_{B} \xi_{A}, A, B=1,2, \cdots, L$, see References [8] and [12]. The Grassman algebra is the direct sum of even and odd sub-algebras: $\mathcal{B}_{L}=\mathcal{B}_{L}^{e}+\mathcal{B}_{L}^{o}$.

\section{1 $\mathcal{N}=2$ super-time and configuration super-space}

The evolution is characterized by the $\mathcal{N}=2$ super-time which is the $\mathbb{R}^{1 \mid 2}$ super-manifold in the terminology of Reference [30]. A given "super-instant" $\left(t, \tau^{1}, \tau^{2}\right)$ is determined by the even, $t \in \mathcal{B}_{L}^{e}$, and odd, $\tau^{\alpha} \in \mathcal{B}_{L}^{o}, \alpha=1,2$, parameters, satisfying the commutation/anti-commutation relations:

$$
\left[t, \tau^{\alpha}\right]=0 \quad, \quad\left\{\tau^{\alpha}, \tau^{\beta}\right\}=0 \quad .
$$

The $\mathcal{N}=2$ configuration super-space is the $\mathcal{C}=\mathbb{R}^{2 \mid 4}$ super-manifold. A super-point in $\mathcal{C}$ is determined by the coordinates $\left(x^{j}, \theta_{\alpha}^{j}\right), j=1,2, \alpha=1,2$, satisfying the commutation/anticommutation rules:

$$
\left[x^{j}, \theta_{\alpha}^{k}\right]=0 \quad, \quad\left\{\theta_{\alpha}^{j}, \theta_{\beta}^{k}\right\}=0 \quad, \quad\left\{\theta_{\alpha}^{j}, \tau^{\beta}\right\}=0 \quad .
$$

Thus, $\left(x^{1}, x^{2}, \theta_{1}^{1}, \theta_{2}^{1}, \theta_{1}^{2}, \theta_{2}^{2}\right) \in \mathcal{B}_{L}^{e} \times \mathcal{B}_{L}^{e} \times \mathcal{B}_{L}^{o} \times \mathcal{B}_{L}^{o} \times \mathcal{B}_{L}^{o} \times \mathcal{B}_{L}^{o}$ and a "super-path"

$$
X^{j}\left(t, \tau^{1}, \tau^{2}\right): \mathbb{R}^{1 \mid 2} \longrightarrow \mathbb{R}^{2 \mid 4}
$$

in $\mathcal{C}$ is given in terms of its components as:

$$
X^{j}\left(t, \tau^{1}, \tau^{2}\right)=x^{j}(t)+\theta_{\alpha}^{j}(t) \tau^{\alpha}+i F^{j}(t) \tau^{1} \tau^{2}
$$

The $F^{j}(t)$ components of the super-path are needed to match the number of "bosonic" (even), $x^{j}, F^{j}$, and "fermionic" (odd), $\theta_{\alpha}^{j}$, degrees of freedom.

Besides the time-translation invariance generated by the Hamiltonian operator $\tilde{H}=-i \partial_{t}$, we seek a super-dynamics that is also invariant under the two left super-time-translations:

$$
\begin{array}{llll}
\text { supersymmetry } 1: & \tau^{1} \rightarrow \tau^{1}-\varepsilon^{1} & \tau^{2} \rightarrow \tau^{2} & t \rightarrow t-i \tau^{1} \varepsilon^{1} \\
\text { supersymmetry 2: } & \tau^{1} \rightarrow \tau^{1} & \tau^{2} \rightarrow \tau^{2}-\varepsilon^{2} & t \rightarrow t-i \tau^{2} \varepsilon^{2}
\end{array}
$$


where $\varepsilon^{\alpha}$ is an infinitesimal odd parameter. The generators of these transformations are the super-charges,

$$
\tilde{Q}_{1}=i \tau^{1} \partial_{t}-\partial_{1} \quad, \quad \tilde{Q}_{2}=i \tau^{2} \partial_{t}-\partial_{2} \quad, \quad \partial_{\alpha}=\frac{\partial}{\partial \tau^{\alpha}} \quad,
$$

that close the $\mathcal{N}=2$ super-algebra

$$
\left\{\tilde{Q}_{\alpha}, \tilde{Q}_{\beta}\right\}=2 \delta_{\alpha \beta} \tilde{H} \quad, \quad\left[\tilde{Q}_{\alpha}, \tilde{H}\right]=0 \quad, \quad[\tilde{H}, \tilde{H}]=0
$$

with the Hamiltonian. The action of the super-charges on a super-path expressed on the component paths is:

$$
\begin{gathered}
\text { Supersymmetry } 1 \\
\delta_{1} X^{j}=\varepsilon \tilde{Q}_{1} X^{j} \Rightarrow\left\{\begin{array}{l}
\delta_{1} x^{j}=\varepsilon \theta_{1}^{j} \\
\delta_{1} \theta_{1}^{j}=i \varepsilon \dot{x}^{j} \\
\delta_{1} \theta_{2}^{j}=-i \varepsilon F^{j} \\
\delta_{1} F^{j}=-\varepsilon \dot{\theta}_{2}^{j}
\end{array} \quad \delta_{2} X^{j}=\varepsilon \tilde{Q}_{2} X^{j} \Rightarrow\left\{\begin{array}{l}
\delta_{2} x^{j}=\varepsilon \theta_{2}^{j} \\
\delta_{2} \theta_{1}^{j}=i \varepsilon F^{j} \\
\delta_{2} \theta_{2}^{j}=i \varepsilon \dot{x}^{j} \\
\delta_{2} F^{j}=\varepsilon \dot{\theta}_{1}^{j}
\end{array}\right.\right.
\end{gathered}
$$

The generators $D_{\alpha}=i \tau^{\alpha} \partial_{t}+\partial_{\alpha}$ of right super-time-translations are usually called covariant derivatives because $\left\{\tilde{Q}_{\alpha}, D_{\beta}\right\}=0$ and:

$$
\delta_{\beta} D_{\alpha} X^{j}=\varepsilon \tilde{Q}_{\beta} D_{\alpha} X^{j}=D_{\alpha} \delta_{\beta} X^{j}
$$

From the free super-Lagrangian $\mathcal{L}_{0}$

$$
\begin{aligned}
\mathcal{L}_{0}\left[x^{j}, \theta_{\alpha}^{j}, F^{j}\right]= & \frac{1}{4} \varepsilon^{\alpha \beta} D_{\alpha} X^{j} D_{\beta} X^{j} \\
= & \frac{1}{4} \theta_{1}^{j} \theta_{2}^{j}-\frac{i}{2} \tau^{1}\left(\dot{x}^{j} \theta_{2}^{j}+\theta_{1}^{j} F^{j}\right) \\
& +\frac{i}{2} \tau^{2}\left(\dot{x}^{j} \theta_{1}^{j}-\theta_{2}^{j} F^{j}\right)+\tau^{2} \tau^{1}\left(\frac{1}{2} \dot{x}^{j} \dot{x}^{j}+\frac{i}{2} \theta_{\alpha}^{j} \dot{\theta}_{\alpha}^{j}+\frac{1}{2} F^{j} F^{j}\right)
\end{aligned}
$$

and the "super-potential", a function $W\left[X^{j}\left(t, \tau^{1}, \tau^{2}\right)\right]$ of the super-path,

$$
W\left[X^{j}\left(t, \tau^{1}, \tau^{2}\right)\right]=W\left[x^{j}(t)\right]-\tau^{\alpha} \theta_{\alpha}^{j} \frac{\partial W}{\partial x^{j}}+\tau^{1} \tau^{2}\left(i F^{j} \frac{\partial W}{\partial x^{j}}-\frac{\partial^{2} W}{\partial x^{j} \partial x^{k}} \theta_{1}^{j} \theta_{2}^{k}\right),
$$

the "super-action" is built:

$$
S=\int d t d \tau^{1} d \tau^{2}\left[\frac{1}{4} \varepsilon^{\alpha \beta} D_{\alpha} X^{j} D_{\beta} X^{j}+i W\left[X^{j}\right]\right]
$$

which is invariant under the two left super-time-translations. Here, $\varepsilon^{\alpha \beta}$ is the completely antisymmetric symbol: $\varepsilon^{12}=-\varepsilon^{21}=1, \varepsilon^{\alpha \alpha}=0$. To check that the transformations generated by $\tilde{Q}_{\alpha}$ are "super-symmetries" of $S$ is not difficult:

$$
\delta_{\alpha} \mathcal{L}_{0}=\varepsilon \tilde{Q}_{\alpha} \mathcal{L}_{0}=\varepsilon\left(i \tau^{\alpha} \partial_{t}-\partial_{\alpha}\right) \mathcal{L}_{0} \quad, \quad \delta_{\alpha} W=\varepsilon \tilde{Q}_{\alpha} W=\varepsilon\left(i \tau^{\alpha} \partial_{t}-\partial_{\alpha}\right) W
$$


Both $i \varepsilon \tau^{\alpha} \partial_{t} \mathcal{L}_{0}$ and $i \varepsilon \tau^{\alpha} \partial_{t} W$ are exact time derivatives and as such do not contribute to variations of $S . \partial_{\alpha} \mathcal{L}_{0}$ and $\partial_{\alpha} W$ are at most linear in $\tau^{\beta}$. The Berezin integration measure on odd Grassman variables,

$$
\int d \tau^{\alpha}=0 \quad, \quad \int d \tau^{\alpha} \tau^{\beta}=\delta^{\alpha \beta},
$$

tells us that $\int d \tau^{1} d \tau^{2} \partial_{\alpha} \mathcal{L}_{0}=\int d \tau^{1} d \tau^{2} \partial_{\alpha} W=0$.

\subsection{Lagrangian and Hamiltonian formalism in Euclidean $\mathbb{R}^{2 / 4}$}

Berezin integration in $S$, (4), plus use of the constraint equations $F^{j}=\frac{\partial W}{\partial x^{j}}$ to eliminate the auxiliary bosonic variables lead us to the supersymmetric Lagrangian:

$$
L=\frac{1}{2} \dot{x}^{j} \dot{x}^{j}+\frac{i}{2} \theta_{a}^{j} \dot{\theta}_{a}^{j}-\frac{1}{2} \frac{\partial W}{\partial x^{j}} \frac{\partial W}{\partial x^{j}}-i \frac{\partial^{2} W}{\partial x^{j} \partial x^{k}} \theta_{1}^{j} \theta_{2}^{k}
$$

The Lagrangian is defined on even elements of $\mathcal{C}$. Besides the natural Lagrangian on the bosonic degrees of freedom with a positive semi-definite potential term, the Grassmannian kinetic energy and a Yukawa coupling between bosonic and fermionic degrees of freedom enter to guarantee supersymmetry. The necessary and sufficient condition for extending classical mechanical systems to the supersymmetric framework is therefore:

$$
U\left(x_{1}, x_{2}\right)=\frac{1}{2} \frac{\partial W}{\partial x^{j}} \frac{\partial W}{\partial x^{j}}
$$

The potential energy $U\left(x^{j}\right)$ is equal to the square of the norm of the gradient of the superpotential.

The Euler-Lagrange equations are:

$$
\ddot{x}^{k}+\frac{\partial U}{\partial x^{k}}+i \frac{\partial^{3} W}{\partial x^{j} \partial x^{l} \partial x^{k}} \theta_{1}^{j} \theta_{2}^{l}=0 \quad \dot{\theta}_{1}^{i}=\frac{\partial^{2} W}{\partial x^{i} \partial x^{j}} \theta_{2}^{j} \quad \dot{\theta}_{2}^{i}=-\frac{\partial^{2} W}{\partial x^{j} \partial x^{i}} \theta_{1}^{j} \quad . .
$$

Looking at the first formula in (7), we notice that even though the bosonic variables were real ordinary magnitudes at the initial time, the evolution of the system would convert them into Grassmannian even variables.

Nöether's theorem dictates that the Hamiltonian functions associated to the vector fields $\tilde{H}, \tilde{Q}_{1}, \tilde{Q}_{2}$ are respectively:

$$
\begin{aligned}
H & =\frac{1}{2} \dot{x}^{j} \dot{x}^{j}+\frac{1}{2} \frac{\partial W}{\partial x^{j}} \frac{\partial W}{\partial x^{j}}+i \frac{\partial^{2} W}{\partial x^{j} \partial x^{k}} \theta_{1}^{j} \theta_{2}^{k} \\
Q_{1} & =\dot{x}^{j} \theta_{1}^{j}-\frac{\partial W}{\partial x^{j}} \theta_{2}^{j} \quad Q_{2}=\dot{x}^{j} \theta_{2}^{j}+\frac{\partial W}{\partial x^{j}} \theta_{1}^{j}
\end{aligned}
$$

$H, Q_{1}$ and $Q_{2}$ are thus first-integrals for the system of ODE (7).

We shall now briefly discuss the Hamiltonian formalism [9], in order to describe how $H, Q_{1}$ and $Q_{2}$ induce the flows associated to the time- and super-time-translations in the co-tangent 
bundle to the configuration super-space. The usual definition of generalized momentum $p_{j}=\frac{\partial L}{\partial \dot{x}^{j}}$ is extended to the Grassmannian variables as follows:

$$
\pi_{\theta_{\alpha}^{j}}=L \frac{\overleftarrow{\partial}}{\partial \dot{\theta}_{\alpha}^{j}}=\frac{i}{2} \theta_{\alpha}^{j}
$$

There is a 12 -dimensional phase space $T^{*} \mathcal{C}$ with local coordinates $\left(x^{j}, \theta_{\alpha}^{j}, p_{j}, \pi_{\theta_{\alpha}^{j}}\right)$. Note, however, the dependence of the fermionic generalized momenta on the Grassman coordinates, coming from the fact that the Grassman kinetic energy is of first-order in time derivatives. The associated four second-class constraints are enforced through Grassmann Lagrange multipliers $\lambda_{a}^{j},[10]$ :

$$
H_{T}=\frac{1}{2} p_{j} p_{j}+\frac{1}{2} \frac{\partial W}{\partial x^{j}} \frac{\partial W}{\partial x^{j}}+i \frac{\partial^{2} W}{\partial x^{j} \partial x^{k}} \theta_{1}^{j} \theta_{2}^{k}-\left(\pi_{\theta_{a}^{j}}-\frac{i}{2} \theta_{a}^{j}\right) \lambda_{a}^{j}
$$

The motion equations are of the canonical form:

$$
\dot{x}^{j}=\frac{\partial H_{T}}{\partial p_{j}} \quad \dot{p}_{j}=-\frac{\partial H_{T}}{\partial x^{j}} \quad \dot{\theta}_{\alpha}^{j}=\frac{\partial H_{T}}{\partial \pi_{\theta_{\alpha}^{i}}} \quad \dot{\pi}_{\theta_{\alpha}^{j}}=\frac{\partial H_{T}}{\partial \theta_{\alpha}^{j}}
$$

Note should be taken of the difference in sign between the bosonic and fermionic canonical equations. Using the constraint equations $\frac{\partial H_{T}}{\partial \lambda_{\alpha}^{j}}=0$, we write

$$
H_{T}=\frac{1}{2} p_{j} p_{j}+\frac{1}{2} \frac{\partial W}{\partial x^{j}} \frac{\partial W}{\partial x^{j}}-\frac{\partial^{2} W}{\partial x^{j} \partial x^{k}}\left(\pi_{\theta_{2}^{k}} \theta_{1}^{j}-\pi_{\theta_{1}^{j}} \theta_{2}^{k}\right)
$$

to rule the right Hamiltonian flow in the phase space.

The Poisson brackets for two generic functions $F$ and $G$ are also generalized to the Grassman variables:

$$
\{F, G\}_{P}=\frac{\partial F}{\partial p_{j}} \frac{\partial G}{\partial q^{j}}-\frac{\partial F}{\partial q^{j}} \frac{\partial G}{\partial p_{j}}+i F \frac{\overleftarrow{\partial}}{\partial \theta_{\alpha}^{j}} \frac{\vec{\partial}}{\partial \theta_{\alpha}^{j}} G-\frac{1}{2} F \frac{\overleftarrow{\partial}}{\partial \pi_{\theta_{\alpha}^{j}}} \frac{\vec{\partial}}{\partial \theta_{\alpha}^{j}} G-\frac{1}{2} F \frac{\overleftarrow{\partial}}{\partial \theta_{\alpha}^{j}} \frac{\vec{\partial}}{\partial \pi_{\theta_{\alpha}^{j}}} G-\frac{i}{4} F \frac{\overleftarrow{\partial}}{\partial \pi_{\theta_{\alpha}^{j}}} \frac{\vec{\partial}}{\partial \pi_{\theta_{\alpha}^{j}}} G
$$

and the canonical equations are of the form

$$
\frac{d f}{d t}=\left\{H_{T}, f\right\}
$$

for any $f=x^{j}, \theta_{\alpha}^{j}, p_{j}, \pi_{\theta_{\alpha}^{j}}$. In general, the time dependence of any observable $F$ is determined by the Poisson structure: $\frac{d F}{d t}=\left\{H_{T}, F\right\}_{P}$. Therefore, the constants of motion, i.e. the invariants, are the physical observables complying with the relationship $\left\{H_{T}, F\right\}_{P}=0$.

In practical terms, it is better to work on the reduced (eight dimensional) phase space. The reduced Hamiltonian is

$$
H=\frac{1}{2} p_{j} p_{j}+\frac{1}{2} \frac{\partial W}{\partial x^{j}} \frac{\partial W}{\partial x^{j}}+i W_{j k} \theta_{1}^{j} \theta_{2}^{k} \quad, \quad W_{j k}=\frac{\partial^{2} W}{\partial x^{j} \partial x^{k}} \quad,
$$

whereas the reduced Poisson brackets

$$
\{F, G\}_{P}=\frac{\partial F}{\partial p_{j}} \frac{\partial G}{\partial q^{j}}-\frac{\partial F}{\partial q^{j}} \frac{\partial G}{\partial p_{j}}+i F \frac{\partial}{\partial \theta_{\alpha}^{j}} \frac{\partial}{\partial \theta_{\alpha}^{j}} G
$$


are obtained from the following Poisson structure:

$$
\left\{p_{j}, x^{k}\right\}_{P}=\delta_{j}^{k} \quad\left\{x^{j}, x^{k}\right\}_{P}=\left\{p_{j}, p_{k}\right\}_{P}=0 \quad\left\{\theta_{\alpha}^{j}, \theta_{\beta}^{k}\right\}_{P}=i \delta^{j k} \delta_{\alpha \beta} \quad .
$$

Also, the canonical equations and the invariant observables must be referred to the reduced Hamiltonian $H$. The most remarkable feature of the super-charges

$$
Q_{1}=p_{j} \theta_{1}^{j}-\frac{\partial W}{\partial x^{j}} \theta_{2}^{j} \quad Q_{2}=p_{j} \theta_{2}^{j}+\frac{\partial W}{\partial x^{j}} \theta_{1}^{j}
$$

is seen through the Poisson structure:

$$
\begin{aligned}
\left\{Q_{1}, Q_{1}\right\}_{P}=2 H & \left\{Q_{2}, Q_{2}\right\}_{P}=2 H \\
\left\{Q_{\alpha}, H\right\}_{P}=0 & \left\{Q_{1}, Q_{2}\right\}_{P}=-i p_{j} \frac{\partial W}{\partial x^{j}} .
\end{aligned}
$$

The Hamiltonian functions $Q_{\alpha}$ and $H$ close a central extension of the $\mathcal{N}=2$ SUSY algebra (1) by a topological term: $Z=-i p_{j} \frac{\partial W}{\partial x^{j}}$ is a total derivative with physical implications only if non-trivial boundary conditions or a non-trivial topology of the configuration superspace are considered. The flow generated by $Q_{\alpha}$ in the co-tangent bundle to the configuration super-space,

\section{Supersymmetry 1}

$$
\begin{aligned}
& \delta_{1} x^{j}=\varepsilon\left\{Q_{1}, x^{j}\right\}_{P}=\varepsilon \theta_{1}^{j} \\
& \delta_{1} \theta_{1}^{j}=\varepsilon\left\{Q_{1}, x^{j}\right\}_{P}=i \varepsilon p_{j} \\
& \delta_{1} \theta_{2}^{j}=\varepsilon\left\{Q_{1}, x^{j}\right\}_{P}=-i \varepsilon \frac{\partial W}{\partial x^{j}}
\end{aligned}
$$

Supersymmetry 2

$$
\begin{aligned}
& \delta_{2} x^{j}=\varepsilon\left\{Q_{2}, x^{j}\right\}_{P}=\varepsilon \theta_{2}^{j} \\
& \delta_{2} \theta_{1}^{j}=\varepsilon\left\{Q_{2}, x^{j}\right\}_{P}=i \varepsilon \frac{\partial W}{\partial x^{j}} \\
& \delta_{2} \theta_{2}^{j}=\varepsilon\left\{Q_{2}, x^{j}\right\}_{P}=i \varepsilon p_{j}
\end{aligned}
$$

represents the two odd super-time translations. Thus, there is a bosonic invariant, the Hamiltonian $H$ itself, due to invariance of the theory with respect to even super-time translations. There are also two fermionic constants of motion, the super-charges $Q_{1}$ and $Q_{2}$ - their Poisson bracket with $H$ is zero- showing the invariance of the system with respect odd super-time translations.

As in every $\mathcal{N}=2$ super-symmetric theory, there is an $R$-symmetry with respect to rotations of the components of the fermionic variables. In our system, $\theta_{\alpha}^{j}$ can be understood as Grassman Majorana spinors and one checks that the reduced Hamiltonian is invariant under the $R$ transformation:

$$
\tilde{\theta}_{1}^{j}=\cos \omega \theta_{1}^{j}+\sin \omega \theta_{2}^{j} \quad, \quad \tilde{\theta}_{2}^{j}=-\sin \omega \theta_{1}^{j}+\cos \omega \theta_{2}^{j} .
$$

The infinitesimal generator of this fermionic symmetry is the, quadratic in the odd coordinates but bosonic, invariant $S_{2}=i \theta_{1}^{j} \theta_{2}^{j}$, see Reference [12]:

$$
\left\{S_{2}, \theta_{1}^{j}\right\}_{P}=\theta_{2}^{j} \quad, \quad\left\{S_{2}, \theta_{2}^{j}\right\}_{P}=-\theta_{1}^{j} \quad .
$$

It was pointed out by Plyushchay, [17], that there is another bosonic invariant, $S_{3}=\prod_{i=1}^{N} \theta_{1}^{i} \theta_{2}^{i}$ when the number of degrees of freedom is $N$. If $N=2, S_{3}=\theta_{1}^{1} \theta_{2}^{1} \theta_{1}^{2} \theta_{2}^{2}=-\frac{1}{2} S_{2}^{2}$ and also comes from the $R$-symmetry. The main goal of this work is to study what happens with super-symmetric extensions in integrable bosonic dynamical systems, where more invariants than the Hamiltonian exist. 


\subsection{Supersymmetric classical mechanics on Riemannian manifolds}

If the bosonic piece of the configuration super-space is a general Riemannian manifold $M^{2}$ equipped with a metric tensor $g_{i j}$, the Grassman variables $\vartheta_{\alpha}^{j}$ under a local change of coordinates $x^{j} \rightarrow \tilde{x}^{j}$ changes as: $\tilde{\vartheta}_{\alpha}^{j}=\frac{\partial \tilde{x}^{j}}{\partial x^{k}} \vartheta_{\alpha}^{k}$.

If the "body" of the configuration super-space is a two dimensinal Riemannian manifold the $\mathcal{N}=2$ super-symmetric action reads:

$$
S=\int d t d \tau^{1} d \tau^{2}\left\{\frac{1}{4} g_{j k}\left(X^{l}\right) \epsilon^{\alpha \beta} D_{\alpha} X^{j} D_{\beta} X^{k}+i W\left[X^{j}\right]\right\} \quad .
$$

The expansion of the super-path in the super-time

$$
X^{j}\left[t, \tau^{1}, \tau^{2}\right]=x^{j}(t)+\vartheta_{\alpha}^{j} \tau^{\alpha}+i F^{j}(t) \tau^{1} \tau^{2}
$$

the metric tensor,

$$
g_{j k}\left(X^{l}\right)=g_{j k}\left(x^{l}\right)+\frac{\partial g_{j k}}{\partial x^{l}} \vartheta_{\alpha}^{l} \tau^{\alpha}+\tau^{1} \tau^{2}\left(i \frac{\partial g_{j k}}{\partial x^{l}} F^{l}-\frac{\partial^{2} g_{j k}}{\partial x^{r} \partial x^{s}} \vartheta_{1}^{r} \vartheta_{2}^{s}\right)
$$

and the super-potential,

$$
\begin{array}{cc}
W\left[X^{j}\left(t, \tau^{1}, \tau^{2}\right)\right]= & W\left[x^{j}(t)\right]-\tau^{\alpha} \theta_{\alpha}^{j} W_{; j}+\tau^{1} \tau^{2}\left(i F^{j} W_{; j}-W_{; j ; k} \theta_{1}^{j} \theta_{2}^{k}\right) \\
W_{; j}=\frac{\partial W}{\partial x^{j}} \quad, \quad & W_{; j ; k}=\frac{\partial^{2} W}{\partial x^{j} \partial x^{k}}-\Gamma_{j k}^{l} \frac{\partial W}{\partial x^{l}}
\end{array}
$$

are richer than for flat manifolds. $\Gamma_{j k}^{l}$ are the Christoffel symbols.

Berezin integration and use of the constraint equations for the auxiliary fields leads to the super-symmetric action:

$$
S=\int d t\left\{\frac{1}{2} g_{j k} \dot{x}^{j} \dot{x}^{k}+\frac{i}{2} g_{j k} \vartheta_{\alpha}^{j} D_{t} \vartheta_{\alpha}^{k}+\frac{1}{4} R_{j k l n} \vartheta_{1}^{j} \vartheta_{2}^{l} \vartheta_{1}^{k} \vartheta_{2}^{n}-\frac{1}{2} g^{j k} \frac{\partial W}{\partial x^{j}} \frac{\partial W}{\partial x^{k}}-i W_{; j ; k} \vartheta_{1}^{j} \vartheta_{2}^{k}\right\}
$$

where

$$
D_{t} \vartheta_{\alpha}^{j}=\dot{\vartheta}_{\alpha}^{j}+\Gamma_{s r}^{j} \dot{x}^{r} \vartheta_{\alpha}^{s}
$$

is the covariant derivative for the Grassman variables, $R_{j k l n}$ is the curvature tensor of the metric, and

$$
U\left(x^{j}\right)=\frac{1}{2} g^{j k} \frac{\partial W}{\partial x^{j}} \frac{\partial W}{\partial x^{k}} .
$$

Nöther's theorem applied to the invariance of the action with respect to the transformation,

$$
\delta_{1} x^{j}=\varepsilon \vartheta_{1}^{j} \quad, \quad \delta_{1} \vartheta_{1}^{j}=i \varepsilon \dot{x}^{j} \quad, \quad \delta_{1} \vartheta_{2}^{j}=-i \varepsilon\left(g^{j k} \frac{\partial W}{\partial x^{k}}-i \Gamma_{k l}^{j} \vartheta_{1}^{j} \vartheta_{2}^{k}\right),
$$

gives the conserved super-charge:

$$
Q_{1}=g_{j k} \dot{x}^{j} \vartheta_{1}^{k}-\frac{\partial W}{\partial x^{j}} \vartheta_{2}^{j}
$$


Invariance with respect to

$$
\delta_{2} x^{j}=\varepsilon \vartheta_{2}^{j} \quad, \quad \delta_{2} \vartheta_{1}^{j}=i \varepsilon\left(g^{j k} \frac{\partial W}{\partial x^{k}}-i \Gamma_{k l}^{j} \vartheta_{1}^{j} \vartheta_{2}^{k}\right) \quad, \quad \delta_{2} \vartheta_{2}^{j}=i \varepsilon \dot{x}^{j}
$$

leads to the second conserved super-charge:

$$
Q_{2}=g_{j k} \dot{x}^{j} \vartheta_{2}^{k}+\frac{\partial W}{\partial x^{j}} \vartheta_{1}^{j}
$$

The Hamiltonian is:

$$
H=\frac{1}{2} g_{j k} \dot{x}^{j} \dot{x}^{k}+\frac{1}{2} g^{j k} \frac{\partial W}{\partial x^{j}} \frac{\partial W}{\partial x^{k}}+i W_{; j ; k} \theta_{1}^{j} \theta_{2}^{k}
$$

\section{Integrability versus supersymmetry: from Liouville to SuperLiouville Models}

An $N$-dimensional Hamiltonian system is said to be completely integrable in the sense of ArnoldLiouville if there exist $N$ integrals of motion, $I_{1}, \cdots, I_{N}$, which are in involution; i.e.,

$$
\left\{I_{j}, I_{k}\right\}_{P}=0 \quad, j, k=1,2, \cdots, N,
$$

see e.g. [31]. In practical terms, explicit integration of the motion equations is more accessible if the Hamilton-Jacobi equation is separable in some appropriate system of coordinates. In such a case, a complete solution of the Hamilton-Jacobi equation is available that, in turn, provides explicit formulas for the trajectories via the Hamilton-Jacobi method. Choosing $I_{1}$ as the Hamiltonian, the time-independent Hamilton-Jacobi equation for zero energy $I_{1}\left(p_{j}, x^{j}\right)=i_{1}=0$ and $V=-U$ is no more than the PDE (11). Thus, the body of the superpotential is the Hamilton's characteristic function for a natural dynamical system with potential $V=-U: S\left(x^{j}, t\right)=W\left(x^{j}\right)-i_{1} t$. A supersymmetric mechanical system is built with a solution of the associated Hamilton-Jacobi equation from the start.

For a special type of completely integrable system, termed as Hamilton-Jacobi-separable, the Hamilton-Jacobi equation (11) becomes equivalent to a system of $N$ non-coupled ODE's. Liouville systems [15] are $N=2$ Hamilton-Jacobi-separable systems of the form: $L=\frac{1}{2}\left(g_{11}\left(x^{1}, x^{2}\right) \dot{x}^{1} \dot{x}^{1}+\right.$ $\left.g_{22}\left(x^{1}, x^{2}\right) \dot{x}^{2} \dot{x}^{2}\right)-V\left(x^{1}, x^{2}\right)$. The complete solution of (11) consists of $2^{N}$ independent solutions coming from the combinations of the solutions of $N$ one-dimensional problems. In this Section we shall describe the different $2^{N}$ supersymmetric extensions of classical Hamilton-Jacobi separable models obtained from the distinct $2^{N}$ Hamilton's characteristic functions, in the special case of Liouville models [15].

\subsection{Liouville models}

Liouville models are two-dimensional completely integrable natural systems with dynamics governed by Lagrangians of the form:

$$
L=\frac{1}{2}\left(g_{11}\left(x^{1}, x^{2}\right) \dot{x}^{1} \dot{x}^{1}+g_{22}\left(x^{1}, x^{2}\right) \dot{x}^{2} \dot{x}^{2}\right)-V\left(x^{1}, x^{2}\right)
$$


The key property enjoyed by this class of systems is that the Hamilton-Jacobi equation for them is separable using two-dimensional elliptic, polar, parabolic or Cartesian systems of coordinates. There are four types classified by the kind of system of coordinates suitable for solving the HJ equation and the two classical invariants in involution are well known for each Liouville system. We briefly describe the four possibilities:

- Liouville Models of Type I: Let us consider the map $\xi: \mathbb{R}^{2} \longrightarrow \mathbb{D}^{2}$, where $\mathbb{D}^{2}$ is an open sub-set of $\mathbb{R}^{2}$ with coordinates $(u, v)$, and let $\xi^{-1}: \mathbb{D}^{2} \longrightarrow \mathbb{R}^{2}$ be the inverse map:

$$
\begin{gathered}
\left(x^{1}, x^{2}\right)=\xi^{-1}(u, v)=\left(\frac{1}{c} u v, \pm \frac{1}{c} \sqrt{\left(u^{2}-c^{2}\right)\left(c^{2}-v^{2}\right)}\right) \\
\xi\left(x^{1}, x^{2}\right)=(u, v) \\
u=\frac{1}{2}\left(\sqrt{\left(x^{1}+c\right)^{2}+x^{2} x^{2}}+\sqrt{\left(x^{1}-c\right)^{2}+x^{2} x^{2}}\right) \\
v= \pm \frac{1}{2}\left(\sqrt{\left(x^{1}+c\right)^{2}+x^{2} x^{2}}-\sqrt{\left(x^{1}-c\right)^{2}+x^{2} x^{2}}\right)
\end{gathered}
$$

The $u, v$ variables are the elliptic coordinates of the bosonic system: $u \in[c, \infty), v \in[-c, c]$ and $\mathbb{D}^{2}$ is the closure of the infinite strip: $\overline{\mathbb{D}}^{2}=[c, \infty) \times[-c, c]$. Let us assume the notation $\xi^{*}$ for the induced map in the functions on $\mathbb{R}^{2}$, i.e. $\xi^{*} U\left(x^{1}, x^{2}\right)=U\left(\xi\left(x^{1}, x^{2}\right)\right) \equiv U(u, v)$, so we will write $U$ for $U\left(x^{1}, x^{2}\right)$ and $\xi^{*} U$ for $U(u, v)$ and a similar convention will be used for the functions in the phase and co-phase spaces.

In the new variables the Lagrangian of a Liouville model of Type I is constrained to be of the following form:

$$
\xi^{*} L=\frac{1}{2} \frac{u^{2}-v^{2}}{u^{2}-c^{2}} \dot{u} \dot{u}+\frac{1}{2} \frac{u^{2}-v^{2}}{c^{2}-v^{2}} \dot{v} \dot{v}-\frac{u^{2}-c^{2}}{u^{2}-v^{2}} f(u)-\frac{c^{2}-v^{2}}{u^{2}-v^{2}} g(v)
$$

where $f(u)$ and $g(v)$ are arbitrary functions. Observe that, apart from a common factor, the contribution to the Lagrangian of the $u$ and $v$ variables splits completely. The HamiltonJacobi equation for zero energy and $V=-U$, formula (11), written in elliptic coordinates reads:

$$
\xi^{*} U=\frac{u^{2}-c^{2}}{u^{2}-v^{2}} f(u)+\frac{c^{2}-v^{2}}{u^{2}-v^{2}} g(v)=\frac{1}{2} \frac{u^{2}-c^{2}}{u^{2}-v^{2}}\left(\frac{d F}{d u}\right)^{2}+\frac{1}{2} \frac{c^{2}-v^{2}}{u^{2}-v^{2}}\left(\frac{d G}{d v}\right)^{2} .
$$

assuming separability: $\xi^{*} W=F(u)+G(v) \Rightarrow \frac{\partial^{2} \xi^{*} W}{\partial u \partial v}=0$.

A complete solution of (14) consists of the four combinations of the two independent onedimensional problems:

$$
\begin{aligned}
F(u) & =\int d u \sqrt{2 f(u)} \quad, \quad G(v)=\int d v \sqrt{2 g(v)} \\
\xi^{*} W^{(a, b)} & =(-1)^{a}\left(\int d u \sqrt{2 f(u)}+(-1)^{b} \int d v \sqrt{2 g(v)}\right) \quad, a, b=0,1 .
\end{aligned}
$$


The image of (14)-(15) in the Cartesian plane,

$$
U\left(x^{1}, x^{2}\right)=\frac{1}{2} \frac{\partial W^{(a, b)}}{\partial x^{j}} \frac{\partial W^{(a, b)}}{\partial x^{j}} \quad, \forall a, b=0,1 \quad,
$$

shows that there are four different superpotentials for the same Type I natural Lagrangian. In Cartesian coordinates the separability condition ${\frac{\partial^{2} \xi^{*} W}{\partial u \partial v}}^{(a, b)}=0$ reads:

$$
x^{1} x^{2}\left(\frac{\partial^{2} W^{(a, b)}}{\partial x^{1} \partial x^{1}}-\frac{\partial^{2} W^{(a, b)}}{\partial x^{2} \partial x^{2}}\right)+\left(x^{2} x^{2}-x^{1} x^{1}+c^{2}\right) \frac{\partial^{2} W^{(a, b)}}{\partial x^{1} \partial x^{2}}+x^{2} \frac{\partial W^{(a, b)}}{\partial x^{1}}-x^{1} \frac{\partial W^{(a, b)}}{\partial x^{2}}=0
$$

The Hamiltonian

$$
I_{1}^{(B)}=\frac{1}{2} p_{j} p_{j}+\frac{1}{2} \frac{\partial W^{(a, b)}}{\partial x^{j}} \frac{\partial W^{(a, b)}}{\partial x^{j}} \quad,
$$

independent of $a, b$, is an obvious integral of motion. In elliptic coordinates it reads:

$$
\xi^{*} I_{1}^{(B)}=\frac{1}{2\left(u^{2}-v^{2}\right)}\left\{\left(u^{2}-c^{2}\right)\left(p_{u}^{2}+2 f(u)\right)+\left(c^{2}-v^{2}\right)\left(p_{v}^{2}+2 g(v)\right)\right\} \quad,
$$

where

$$
p_{u}=\frac{u^{2}-v^{2}}{u^{2}-c^{2}} \dot{u} \quad, \quad p_{v}=\frac{u^{2}-v^{2}}{c^{2}-v^{2}} \dot{v}
$$

The inverse image of the second invariant is:

$$
\begin{aligned}
\xi^{*} I_{2}^{(B)} & =\frac{1}{2}\left[\frac{\left(u^{2}-c^{2}\right)\left(c^{2}-v^{2}\right)}{u^{2}-v^{2}} p_{v}^{2}-\frac{\left(u^{2}-c^{2}\right)\left(c^{2}-v^{2}\right)}{u^{2}-v^{2}} p_{u}^{2}\right] \\
& +\frac{\left(u^{2}-c^{2}\right)\left(c^{2}-v^{2}\right)}{2\left(u^{2}-v^{2}\right)}\left[\left(\frac{d G}{d v}\right)^{2}-\left(\frac{d F}{d u}\right)^{2}\right]
\end{aligned}
$$

The direct image provides the intricate second invariant in involution - $\left\{I_{1}^{(B)}, I_{2}^{(B)}\right\}_{P}=0$ with the Hamiltonian:

$$
I_{2}^{(B)}=\frac{1}{2}\left[\left(x^{2} p_{1}-x^{1} p_{2}\right)^{2}-c^{2} p_{2} p_{2}+\left(x^{2} \frac{\partial W^{(a, b)}}{\partial x^{1}}-x^{1} \frac{\partial W^{(a, b)}}{\partial x^{2}}\right)^{2}-c^{2} \frac{\partial W^{(a, b)}}{\partial x^{2}} \frac{\partial W^{(a, b)}}{\partial x^{2}}\right] .
$$

The remarkable fact is that $I_{2}^{(B)}$ is independent of $a, b$, i.e. the four superpotentials also leads to identical second invariants !, not only to the same Hamiltonian.

In the literature about integrable dynamical systems it is usually stated that the existence of the second invariant obeys a hidden symmetry. The definition of the generalized momenta,

$$
\Pi_{j}^{(a, b)}=p_{j}+i \frac{\partial W^{(a, b)}}{\partial x^{j}}
$$


sheds light on the nature of such (non-linear) symmetries. In terms of the generalized momenta we obtain:

$$
I_{1}^{(B)}=\frac{1}{2}\left[\left|\Pi_{1}^{(a, b)}\right|^{2}+\left|\Pi_{2}^{(a, b)}\right|^{2}\right] \quad, \quad I_{2}^{(B)}=\frac{1}{2}\left[\left|x^{2} \Pi_{1}^{(a, b)}-x^{1} \Pi_{2}^{(a, b)}\right|^{2}-c^{2}\left|\Pi_{2}^{(a, b)}\right|^{2}\right] \quad, \forall a, b \quad,
$$

and well-known invariants with $p_{j}$ replaced by $\Pi_{j}^{(a, b)}$ are recognized. $\Pi_{j}=\frac{\partial\left(L+L_{T}\right)}{\partial \dot{x}^{j}}$ can be understood as the canonical momenta coming from the addition of a complex topological piece

$$
L_{T}=i \dot{x}^{j} \frac{\partial W}{\partial x^{j}}
$$

to the Lagrangian $L$, in agreement with the central extension shown in the SUSY Poisson algebra (8). Note that $I_{1}^{(B)}$ can thus be written à la Bogomolny:

$$
I_{1}^{(B)}=\frac{1}{2} \Pi_{j} \Pi_{j}-i \Pi_{j} \frac{\partial W}{\partial x^{j}}
$$

It is also possible, however, to interpret that the generalized momenta by themselves close another extension, now of the ordinary Poisson algebra:

$$
\left\{x^{j}, x^{k}\right\}_{P}=0 \quad, \quad\left\{x^{j}, \Pi_{k}\right\}_{P}=\delta_{k}^{j} \quad, \quad\left\{\Pi_{j}, \Pi_{k}\right\}_{P}=-2 i \frac{\partial^{2} W}{\partial x^{j} \partial x^{k}} \quad .
$$

Both the first and the second invariants obey symmetries related to (non-linear) transformations generated in the framework of the generalized Poisson structure (18).

\section{- Liouville Models of Type II:}

The type II Liouville models are two-dimensional dynamical systems for which the HamiltonJacobi equation is separable using polar coordinates. The direct $-\zeta: \mathbb{R}^{2} \longrightarrow \mathbb{D}^{2} \simeq \mathbb{R}^{2}-\{0\}$ - and inverse - $\zeta^{-1}: \mathbb{R}^{2}-\{0\} \longrightarrow \mathbb{R}^{2}$ - maps determine the change from polar to Cartesian coordinates and viceversa:

$$
\begin{aligned}
(\rho, \chi) & =\zeta\left(x^{1}, x^{2}\right)=\left(\sqrt{x^{1} x^{1}+x^{2} x^{2}}, \arctan \left(\frac{x^{2}}{x^{1}}\right)\right) \\
\left(x^{1}, x^{2}\right) & =\zeta^{-1}(\rho, \chi)=(\rho \cos \chi, \rho \sin \chi) \quad, \rho \in[0, \infty) ; \chi \in[0,2 \pi) .
\end{aligned}
$$

The Lagrangian of the Liouville models of Type II reads:

$$
\zeta^{*} L=\frac{1}{2} \dot{\rho} \dot{\rho}+\frac{1}{2} \rho^{2} \dot{\chi} \dot{\chi}-f(\rho)-\frac{1}{\rho^{2}} g(\chi) \quad .
$$

Again, besides the metric factor $g_{11}=1, g_{22}=\rho^{2}$, the contributions of $\rho$ and $\chi$ appear completely separated in the Lagrangian. Here, the zero energy time-independent HamiltonJacobi equation (assuming separability) (11) is:

$$
\zeta^{*} U=f(\rho)+\frac{1}{\rho^{2}} g(\chi)=\frac{1}{2}\left(\frac{d F}{d \rho}\right)^{2} \pm \frac{1}{2 \rho^{2}}\left(\frac{d G}{d \chi}\right)^{2} \quad .
$$


The complete solution

$$
\begin{aligned}
F(\rho) & =\int d u \sqrt{2 f(\rho)} \quad, \quad G(\chi)=\int d v \sqrt{2 g(\chi)} \\
\zeta^{*} W^{(a, b)} & =(-1)^{a}\left(\int d \rho \sqrt{2 f(\rho)}+(-1)^{b} \int d \chi \sqrt{2 g(\chi)}\right) \quad a, b=0,1,
\end{aligned}
$$

provides us with four different superpotentials to build supersymmetric extensions:

$$
U\left(x^{1}, x^{2}\right)=\frac{1}{2} \frac{\partial W^{(a, b)}}{\partial x^{j}} \frac{\partial W^{(a, b)}}{\partial x^{j}} \quad, \forall a, b=0,1
$$

In Cartesian coordinates the separability condition ${\frac{\partial^{2} \zeta^{*} W}{\partial \rho \partial \chi}}^{(a, b)}=0$ reads

$$
-x^{1} x^{2}\left(\frac{\partial^{2} W^{(a, b)}}{\partial x^{1} \partial x^{1}}-\frac{\partial^{2} W^{(a, b)}}{\partial x^{2} \partial x^{2}}\right)+\left(x^{1} x^{1}-x^{2} x^{2}\right) \frac{\partial^{2} W^{(a, b)}}{\partial x^{1} \partial x^{2}}+x^{1} \frac{\partial W^{(a, b)}}{\partial x^{2}}-x^{2} \frac{\partial W^{(a, b)}}{\partial x^{1}}=0
$$

The two invariants in involution, written in polar coordinates, are:

$$
\zeta^{*} I_{1}^{(B)}=\frac{1}{2} p_{\rho}^{2}+\frac{1}{2 \rho^{2}} p_{\chi}^{2}+f(\rho)+\frac{1}{\rho^{2}} g(\chi) \quad, \quad \zeta^{*} I_{2}^{(B)}=\frac{1}{2 \rho^{2}} p_{\chi}^{2}+\frac{1}{\rho^{2}} g(\chi)
$$

In Cartesian coordinates they are easily shown to be independent of $a$ and $b$ :

$$
\begin{aligned}
I_{1}^{(B)} & =\frac{1}{2} p_{j} p_{j}+\frac{1}{2} \frac{\partial W^{(a, b)}}{\partial x^{j}} \frac{\partial W^{(a, b)}}{\partial x^{j}}=\frac{1}{2}\left|\Pi_{j}^{(a, b)}\right|\left|\Pi_{j}^{(a, b)}\right| \\
I_{2}^{(B)} & =\frac{1}{2}\left(x^{2} \dot{x}^{1}-x^{1} \dot{x}^{2}\right)^{2}+\frac{1}{2}\left(x^{2} \frac{\partial W^{(a, b)}}{\partial x^{1}}-x^{1} \frac{\partial W^{(a, b)}}{\partial x^{2}}\right)^{2}=\frac{1}{2}\left|x^{2} \Pi_{1}^{(a, b)}-x^{1} \Pi_{2}^{(a, b)}\right|^{2}
\end{aligned}
$$

\section{- Liouville Models of Type III :}

In this type of model, the Hamilton-Jacobi equation is separable using parabolic coordinates. The direct - $\gamma: \mathbb{R}^{2} \longrightarrow \mathbb{H}^{2}$ - and inverse - $\gamma^{-1}: \mathbb{H}^{2} \longrightarrow \mathbb{R}^{2}$ - maps between the half-plane and the plane dictate the change from parabolic to Cartesian coordinates and viceversa:

$$
\begin{aligned}
& (u, v)=\gamma\left(x^{1}, x^{2}\right)=\left( \pm \sqrt{\sqrt{x^{1} x^{1}+x^{2} x^{2}}+x^{1}}, \sqrt{\sqrt{x^{1} x^{1}+x^{2} x^{2}}-x^{1}}\right) \\
& \left(x^{1}, x^{2}\right)=\gamma^{-1}(u, v)=\left(\frac{1}{2}\left(u^{2}-v^{2}\right), u v\right) \\
& \mathbb{H}^{2}=(-\infty, \infty) \times[0, \infty) \quad ; \quad u \in(-\infty, \infty), v \in[0, \infty)
\end{aligned}
$$

A Liouville model of Type III obeys a Lagrangian of the form:

$$
\gamma^{*} L=\frac{1}{2}\left(u^{2}+v^{2}\right)(\dot{u} \dot{u}+\dot{v} \dot{v})-\frac{1}{u^{2}+v^{2}}(f(u)+g(v))
$$


The zero-energy static Hamilton-Jacobi equation

$$
\gamma^{*} U=\frac{1}{u^{2}+v^{2}}(f(u)+g(v))=\frac{1}{u^{2}+v^{2}}\left[\left(\frac{d F}{d u}\right)^{2}+\left(\frac{d G}{d v}\right)^{2}\right]
$$

is solved by the four "separate" superpotentials:

$$
\gamma^{*} W^{(a, b)}=(-1)^{a}\left(\int d u \sqrt{2 f(u)}+(-1)^{b} \int d v \sqrt{2 g(v)}\right) \quad .
$$

The separability condition ${\frac{\partial^{2} W}{\partial v \partial u}}^{(a, b)}=0$ in Cartesian coordinates takes the form:

$$
x^{2}\left(\frac{\partial^{2} W^{(a, b)}}{\partial x^{2} \partial x^{2}}-\frac{\partial^{2} W^{(a, b)}}{\partial x^{1} \partial x^{1}}\right)+2 x^{1} \frac{\partial^{2} W^{(a, b)}}{\partial x^{1} \partial x^{2}}+\frac{\partial W^{(a, b)}}{\partial x^{2}}=0
$$

In parabolic coordinates the two invariants in involution can be chosen as:

$$
\begin{aligned}
& \gamma^{*} I_{1}^{(B)}=\frac{1}{2\left(u^{2}+v^{2}\right)}\left(p_{u}^{2}+p_{v}^{2}\right)+\frac{1}{u^{2}+v^{2}}(f(u)+g(v)) \\
& \gamma^{*} I_{2}^{(B)}=\frac{1}{u^{2}+v^{2}}\left(\frac{u^{2}}{2} p_{v}^{2}-\frac{v^{2}}{2} p_{u}^{2}+u^{2} g(v)-v^{2} f(u)\right)
\end{aligned}
$$

They can easily be translated to Cartesian coordinates, giving the same result for all the values of $a$ and $b$ :

$$
\begin{aligned}
I_{1}^{(B)} & =\frac{1}{2} p_{j} p_{j}+\frac{1}{2} \frac{\partial W^{(a, b)}}{\partial x^{j}} \frac{\partial W^{(a, b)}}{\partial x^{j}}=\frac{1}{2}\left|\Pi_{j}^{(a, b)}\right|\left|\Pi_{j}^{(a, b)}\right| \\
I_{2}^{(B)} & =\left(x^{1} p_{2}-x^{2} p_{1}\right) p_{2}+\left(x^{1} \frac{\partial W^{(a, b)}}{\partial x^{2}}-x^{2} \frac{\partial W^{(a, b)}}{\partial x^{1}}\right) \frac{\partial W^{(a, b)}}{\partial x^{2}} \\
& =\operatorname{Re}\left[\left(x^{2} \Pi_{1}^{(a, b)}-x^{1} \Pi_{2}^{(a, b)}\right) \bar{\Pi}_{2}^{(a, b)}\right] .
\end{aligned}
$$

\section{- Liouville Models of Type IV:}

In the fourth type of Liouville models the dynamics of the two degrees of freedom are completely independent:

$$
L=\frac{1}{2} \dot{x}^{1} \dot{x}^{1}+\frac{1}{2} \dot{x}^{2} \dot{x}^{2}-f\left(x^{1}\right)-g\left(x^{2}\right)
$$

In this case there is no need to change the system of coordinates to solve the HJ equation and it is clear that the four superpotentials are:

$$
W^{(a, b)}\left(x^{1}, x^{2}\right)=(-1)^{a}\left(\int d x^{1} \sqrt{f\left(x^{1}\right)}+(-1)^{b} \int d x^{2} \sqrt{g\left(x^{2}\right)}\right) \quad .
$$


The separability condition is

$$
\frac{\partial^{2} W^{(a, b)}}{\partial x^{1} \partial x^{2}}=0
$$

and the two invariants can be chosen as:

$$
\begin{aligned}
I_{1}^{(B)} & =\frac{1}{2} p_{j} p_{j}+\frac{1}{2} \frac{\partial W^{(a, b)}}{\partial x^{j}} \frac{\partial W^{(a, b)}}{\partial x^{j}}=\frac{1}{2}\left|\Pi_{j}^{(a, b)}\right|\left|\Pi_{j}^{(a, b)}\right| \\
I_{2}^{(B)} & =\frac{1}{2} p_{1} p_{1}+\frac{1}{2} \frac{\partial W^{(a, b)}}{\partial x^{1}} \frac{\partial W^{(a, b)}}{\partial x^{1}}=\frac{1}{2}\left|\Pi_{1}^{(a, b)}\right| \cdot\left|\Pi_{1}^{(a, b)}\right|
\end{aligned} .
$$

\subsection{SuperLiouville models}

The strategy for building $\mathcal{N}=2$ supersymmetric Lagrangian systems with Liouville models as the bosonic sector is clear: simply consider the hybrid non-linear Sigma/Wess-Zumino models of subsection $\S 2.3$ whose target space is the Riemannian manifold $\mathbb{M}^{2}=\overline{\mathbb{D}}^{2}$ equipped with the metric induced by the maps $\xi^{*}, \zeta^{*}$ and $\gamma^{*}$ for Types I, II, and III, and the Euclidean metric for Type IV.

- SuperLiouville Models of Type I: The metric and the Christoffel symbols induced by the map $\xi^{*}$ in $\mathbb{D}^{2}=(c, \infty) \times(-c, c)$ are:

$$
\begin{gathered}
g(u, v)=\left(\begin{array}{cc}
g_{u u}=\frac{u^{2}-v^{2}}{u^{2}-c^{2}} & g_{u v}=0 \\
g_{v u}=0 & g_{v v}=\frac{u^{2}-v^{2}}{c^{2}-v^{2}}
\end{array}\right) \\
g^{-1}(u, v)=\left(\begin{array}{cc}
g^{u u}=\frac{u^{2}-c^{2}}{u^{2}-v^{2}} & g^{u v}=0 \\
g^{v u}=0 & g^{v v}=\frac{c^{2}-v^{2}}{u^{2}-v^{2}}
\end{array}\right) \\
\Gamma_{u u}^{u}=\frac{-u\left(c^{2}-v^{2}\right)}{\left(u^{2}-v^{2}\right)\left(u^{2}-c^{2}\right)} \quad, \quad \Gamma_{v v}^{v}=\frac{v\left(u^{2}-c^{2}\right)}{\left(u^{2}-v^{2}\right)\left(c^{2}-v^{2}\right)}, \quad \Gamma_{u v}^{u}=\Gamma_{v u}^{u}=\frac{-v}{u^{2}-v^{2}} \\
\Gamma_{u u}^{v}=\frac{v\left(c^{2}-v^{2}\right)}{\left(u^{2}-v^{2}\right)\left(u^{2}-c^{2}\right)} \quad, \quad \Gamma_{v v}^{u}=\frac{-u\left(u^{2}-c^{2}\right)}{\left(u^{2}-v^{2}\right)\left(c^{2}-v^{2}\right)}, \quad \Gamma_{u v}^{v}=\Gamma_{v u}^{v}=\frac{u}{u^{2}-v^{2}}
\end{gathered}
$$

Besides the bosonic (even Grassman) variables $u, v$, ruled by Lagrangians of Type I as discussed in the previous sub-Section, there are also fermionic (odd Grassman) Majorana spinors $\vartheta_{\alpha}^{u}, \vartheta_{\alpha}^{v}$ in the system. A supersymmetric two dimensional mechanical system is a super- Liouville model of Type I if the Lagrangian is of the form $\xi^{*} L=\xi^{*} L_{B}+\xi^{*} L_{F}+\xi^{*} L_{B F}$ with:

$$
\begin{gathered}
\xi^{*} L_{B}=\frac{1}{2} g_{u u}(u, v) \dot{u} \dot{u}+\frac{1}{2} g_{v v}(u, v) \dot{v} \dot{v}-\frac{1}{2} g^{u u}(u, v)\left(\frac{d F}{d u}\right)^{2}-\frac{1}{2} g^{v v}(u, v)\left(\frac{d G}{d v}\right)^{2} \\
\xi^{*} L_{F}=\frac{i}{2} g_{u u}(u, v) \vartheta_{\alpha}^{u} D_{t} \vartheta_{\alpha}^{u}+\frac{i}{2} g_{v v}(u, v) \vartheta_{\alpha}^{v} D_{t} \vartheta_{\alpha}^{v}
\end{gathered}
$$


and,

$$
\begin{aligned}
\xi^{*} L_{B F}^{I}= & -i\left[\frac{d^{2} F}{d u^{2}}-\Gamma_{u u}^{u} \frac{d F}{d u}-\Gamma_{u u}^{v} \frac{d G}{d v}\right] \vartheta_{1}^{u} \vartheta_{2}^{u}-i\left[\frac{d^{2} G}{d v^{2}}-\Gamma_{v v}^{u} \frac{d F}{d u}-\Gamma_{v v}^{v} \frac{d G}{d v}\right] \vartheta_{1}^{v} \vartheta_{2}^{v}+ \\
& +i\left[\Gamma_{u v}^{u} \frac{d F}{d u}-\Gamma_{u v}^{v} \frac{d G}{d v}\right]\left(\vartheta_{1}^{u} \vartheta_{2}^{v}+\vartheta_{1}^{v} \vartheta_{2}^{u}\right)
\end{aligned}
$$

The fermionic kinetic energy is encoded in $\xi^{*} L_{F}$ and there are Yukawa terms in $\xi^{*} L_{B F}$ ruling the Bose-Fermi interactions.

- SuperLiouville Models of Type II: Everything is analogous trading elliptic for polar coordinates. The metric and the Christoffel symbols are:

$$
\begin{gathered}
g(\rho)=\left(\begin{array}{cc}
g_{\rho \rho}=1 & g_{\rho \chi}=0 \\
g_{\chi \rho}=0 & g_{\chi \chi}=\rho^{2}
\end{array}\right), \quad g^{-1}(\rho)=\left(\begin{array}{cc}
g^{\rho \rho}=1 & g^{\rho \chi}=0 \\
g^{\chi \rho}=0 & g^{\chi \chi}=\frac{1}{\rho^{2}}
\end{array}\right) \\
\Gamma_{\rho \rho}^{\rho}=\Gamma_{\rho \chi}^{\rho}=\Gamma_{\chi \rho}^{\rho}=\Gamma_{\rho \rho}^{\chi}=\Gamma_{\chi \chi}^{\chi}=0, \quad \Gamma_{\chi \chi}^{\rho}=-\rho, \quad \Gamma_{\rho \chi}^{\chi}=\Gamma_{\chi \rho}^{\chi}=\frac{1}{\rho}
\end{gathered}
$$

Therefore, the Lagrangian of a super-Liouville model of Type II including bosonic $\rho, \chi$ and fermionic $\vartheta_{\alpha}^{\rho}, \vartheta_{\alpha}^{\chi}$ variables is the sum of the three pieces:

$$
\begin{gathered}
\zeta^{*} L_{B}=\frac{1}{2} \dot{\rho} \dot{\rho}+\frac{1}{2} g_{\chi \chi} \dot{\chi} \dot{\chi}-\frac{1}{2}\left(\frac{d F}{d \rho}\right)^{2}-\frac{1}{2} g^{\chi \chi}\left(\frac{d G}{d \chi}\right)^{2} \\
\zeta^{*} L_{F}=\frac{i}{2} \vartheta_{\alpha}^{\rho} D_{t} \vartheta_{\alpha}^{\rho}+\frac{i}{2} g_{\chi \chi} \vartheta_{\alpha}^{\chi} D_{t} \vartheta_{\alpha}^{\chi} \\
\zeta^{*} L_{B F}=-i \frac{d^{2} F}{d \rho^{2}} \vartheta_{1}^{\rho} \vartheta_{2}^{\rho}-i\left(\frac{d^{2} G}{d \chi^{2}}-\Gamma_{\chi \chi}^{\rho} \frac{d F}{d \rho}\right) \vartheta_{1}^{\chi} \vartheta_{2}^{\chi}+i \Gamma_{\rho \chi}^{\chi} \frac{d G}{d \chi}\left(\vartheta_{1}^{\rho} \vartheta_{2}^{\chi}+\vartheta_{1}^{\chi} \vartheta_{2}^{\rho}\right)
\end{gathered}
$$

- SuperLiouville Models of Type III: The supersymmetric extensions of Liouville Type III models is easier in parabollic coordinates. The metric and the Christoffel symbols are:

$$
\begin{gathered}
g(u, v)=\left(\begin{array}{cc}
u^{2}+v^{2} & 0 \\
0 & u^{2}+v^{2}
\end{array}\right) \quad, \quad g^{-1}(u, v)=\left(\begin{array}{cc}
\frac{1}{u^{2}+v^{2}} & 0 \\
0 & \frac{1}{u^{2}+v^{2}}
\end{array}\right) \\
\Gamma_{u u}^{u}=\Gamma_{u v}^{v}=\Gamma_{v u}^{v}=-\Gamma_{v v}^{u}=\frac{u}{u^{2}+v^{2}}, \quad \Gamma_{u v}^{u}=\Gamma_{v u}^{u}=\Gamma_{v v}^{v}=-\Gamma_{u u}^{v}=\frac{v}{u^{2}+v^{2}}
\end{gathered}
$$

The dynamics of the SUSY pairs of variables $u, \vartheta_{\alpha}^{u}$ and $v, \vartheta_{\alpha}^{v}$ is governed in a super-Liouville model of Type III by the Lagrangian $\zeta^{*} L=\zeta^{*} L_{B}+\zeta^{*} L_{F}+\zeta^{*} L_{B F}$, where

$$
\begin{gathered}
\zeta^{*} L_{B}=\frac{1}{2} g_{u u} \dot{u} \dot{u}+\frac{1}{2} g_{v v} \dot{v} \dot{v}-\frac{1}{2} g^{u u}\left(\frac{d F}{d u}\right)^{2}+\frac{1}{2} g^{v v}\left(\frac{d G}{d v}\right)^{2}, \\
\zeta^{*} L_{F}=\frac{i}{2} g_{u u} \vartheta_{\alpha}^{u} D_{t} \vartheta_{\alpha}^{u}+\frac{i}{2} g_{v v} \vartheta_{\alpha}^{v} D_{t} \vartheta_{\alpha}^{v}
\end{gathered}
$$


and,

$$
\begin{aligned}
\zeta^{*} L_{B F}= & -i\left[\frac{d^{2} F}{d u^{2}}-\Gamma_{u u}^{u} \frac{d F}{d u}-\Gamma_{u u}^{v} \frac{d G}{d v}\right] \vartheta_{1}^{u} \vartheta_{2}^{u}-i\left[\frac{d^{2} G}{d v^{2}}-\Gamma_{v v}^{u} \frac{d F}{d u}-\Gamma_{v v}^{v} \frac{d G}{d v}\right] \vartheta_{1}^{v} \vartheta_{2}^{v}+ \\
+ & i\left[\Gamma_{u v}^{v} \frac{d G}{d v}+\Gamma_{u v}^{u} \frac{d F}{d u}\right]\left(\vartheta_{1}^{u} \vartheta_{2}^{v}+\vartheta_{1}^{v} \vartheta_{2}^{u}\right)
\end{aligned}
$$

- SuperLiouville Models of Type IV: Finally, the definition of SuperLiouville Model of Type IV is straightforward. The Lagrangian is

$$
L=\frac{1}{2} \dot{x}^{j} \dot{x}^{j}+\frac{i}{2} \theta_{\alpha}^{j} \dot{\theta}_{\alpha}^{j}-\frac{1}{2} \frac{\partial W}{\partial x^{j}} \frac{\partial W}{\partial x^{j}}-i \frac{\partial^{2} W}{\partial x^{1} \partial x^{1}} \theta_{1}^{1} \theta_{2}^{1}-i \frac{\partial^{2} W}{\partial x^{2} \partial x^{2}} \theta_{1}^{2} \theta_{2}^{2}
$$

and the system can be understood as an $(\mathcal{N}=2) \oplus(\mathcal{N}=2)$ SUSY model in $(0+1)$ dimensions.

There is an obvious first integral that can be written in a unified way for all four Types of superLiouville models using Cartesian coordinates:

$$
I_{1}=I_{1}^{(B)}+I_{1}^{(F)} \quad, \quad I_{1}^{(B)}=\frac{1}{2} p_{j} p_{j}+\frac{1}{2} \frac{\partial W^{(a, b)}}{\partial x^{j}} \frac{\partial W^{(a, b)}}{\partial x^{j}} \quad, \quad I_{1}^{(F)}=i \frac{\partial^{2} W^{(a, b)}}{\partial x^{j} \partial x^{k}} \theta_{1}^{j} \theta_{2}^{k} .
$$

$I_{1}^{(B)}$ is formally identical to the Hamiltonian of the parent Liouville model but we stress that $p_{j}$ and $x^{j}$ are now even Grassman variables. It is, in any case, independent of $a$ and $b . I_{1}^{(F)}$ comes from the Yukawa couplings between bosonic and fermionic variables. Note that $I_{1}^{(F)}$ depends on choosing either $b=0$ or $b=1$; thus, each Liouville model admits two different supersymmetric extensions achieved from different solutions of the time-independent Hamilton-Jacobi equation for the Hamilton characteristic function (the superpotential). The choice of $a=1$ instead of $a=0$ changes $I_{1}^{(F)}$ to $-I_{1}^{(F)}$; this flip of sign can be absorbed by exchanging positive and negative energy for the fermionic trajectories.

A final remark is that the separability of the purely bosonic Liouville mechanical systems is lost in the supersymmetric framework because of the Yukawa couplings, except for SuperLiouville models of Type IV.

\section{On the Bosonic Invariants}

Liouville models have a second invariant in involution with the energy -the first invariant- that guarantees complete integrability in the sense of the Liouville theorem. We shall now show that the SuperLiouville models also have a second invariant of bosonic nature. Our strategy in the search for such an invariant, $\{I, H\}_{P}=0$, follows the general pattern found in the literature: see [22]. The ansatz for invariants, at most quadratic in the momenta, is:

$$
\begin{aligned}
I= & \frac{1}{2} H^{i j} p_{i} p_{j}+K\left(x^{1}, x^{2}\right)+F_{i j} \theta_{1}^{i} \theta_{2}^{j}+G_{i j} \theta_{1}^{i} \theta_{1}^{j}+J_{i j} \theta_{2}^{i} \theta_{2}^{j}+ \\
& +L_{j k}^{i} p_{i} \theta_{1}^{j} \theta_{1}^{k}+M_{j k}^{i} p_{i} \theta_{2}^{j} \theta_{2}^{k}+N_{j k}^{i} p_{i} \theta_{1}^{j} \theta_{2}^{k}+S_{i j k l} \theta_{1}^{i} \theta_{2}^{k} \theta_{1}^{j} \theta_{2}^{l}
\end{aligned}
$$

Here, we assume that: 
i) $K$ is a function.

ii) $H^{i j}$ is a symmetric tensor depending on $x^{i}$. There are three independent functions to determine.

iii) $L_{j k}^{i}$ and $M_{j k}^{i}$ also depends only on $x^{i}$ and are antisymmetric in the indices $j$ and $k: L_{j k}^{i}=$ $-L_{k j}^{i}, M_{j k}^{i}=-M_{k j}^{i}$. They include four independent functions.

iv) $G_{i j}$ and $J_{i j}$ are functions, antisymmetric in the indices, of $x^{i}: G_{i j}=-G_{j i}$ and $J_{i j}=-J_{j i}$. A priori $F_{i j}\left(x^{i}\right)$ however, is neither symmetric nor antisymmetric; it contains four independent functions.

$v)$ Finally, $S_{i j k l}\left(x^{i}\right)$ is antisymmetric in the exchange of the indices $i, j$ and $k, l$ and symmetric in the exchange of the pairs $i j, k l$. There is only one independent function to determine in this tensor.

The commutator with the Hamiltonian is:

$$
\begin{aligned}
{[I, H]=} & -\frac{1}{2} \frac{\partial H^{j k}}{\partial x^{l}} p_{l} p_{j} p_{k}+\left(H^{l j} \frac{\partial^{2} W}{\partial x^{j} \partial x^{k}} \frac{\partial W}{\partial x^{k}}-\frac{\partial K}{\partial x^{l}}\right) p_{l}+ \\
& +\left(i H^{n j} \frac{\partial^{3} W}{\partial x^{k} \partial x^{l} \partial x^{j}}-\frac{\partial F_{k l}}{\partial x^{n}}-2 L_{k m}^{n} \frac{\partial^{2} W}{\partial x^{m} \partial x_{l}}-2 M_{l m}^{n} \frac{\partial^{2} W}{\partial x^{m} \partial x^{k}}\right) p_{n} \theta_{1}^{k} \theta_{2}^{l}+ \\
& +\left(-\frac{\partial^{2} W}{\partial x^{l} \partial x^{k}} F_{l j}+M_{k j}^{n} \frac{\partial^{2} W}{\partial x^{n} \partial x^{l}} \frac{\partial W}{\partial x^{l}}\right) \theta_{2}^{k} \theta_{2}^{j}+\left(-\frac{\partial J_{l j}}{\partial x^{k}}-N_{m j}^{k} \frac{\partial^{2} W}{\partial x^{m} \partial x^{l}}\right) p_{k} \theta_{2}^{l} \theta_{2}^{j}- \\
& +\left(\frac{\partial^{2} W}{\partial x^{j} \partial x^{k}} F_{n k}+L_{n j}^{l} \frac{\partial^{2} W}{\partial x^{l} \partial x^{k}} \frac{\partial W}{\partial x^{k}}\right) \theta_{1}^{n} \theta_{1}^{j}+\left(-\frac{\partial G_{n j}}{\partial x^{l}}+N_{n k}^{l} \frac{\partial^{2} W}{\partial x^{j} \partial x^{k}}\right) p_{l} \theta_{1}^{n} \theta_{1}^{j}+ \\
& +2\left(-G_{n j} \frac{\partial^{2} W}{\partial x^{j} \partial x^{k}}-J_{k l} \frac{\partial^{2} W}{\partial x^{n} \partial x^{l}}+\frac{1}{2} N_{n k}^{j} \frac{\partial^{2} W}{\partial x^{j} \partial x^{l}} \frac{\partial W}{\partial x^{l}}\right) \theta_{1}^{n} \theta_{2}^{k}+ \\
& -\frac{\partial L_{j k}^{n}}{\partial x^{l}} p_{l} p_{n} \theta_{1}^{j} \theta_{1}^{k}-\frac{\partial M_{j k}^{n}}{\partial x^{l}} p_{l} p_{n} \theta_{2}^{j} \theta_{2}^{k}-\frac{\partial N_{j k}^{n}}{\partial x^{l}} p_{l} p_{n} \theta_{1}^{j} \theta_{2}^{k}+ \\
+ & i N_{j k}^{n} \frac{\partial^{3} W}{\partial x^{n} \partial x^{l} \partial x^{m}} \theta_{1}^{j} \theta_{2}^{k} \theta_{1}^{l} \theta_{2}^{m}-\frac{\partial S_{n j k l}}{\partial x^{m}} p_{m} \theta_{1}^{n} \theta_{2}^{k} \theta_{1}^{j} \theta_{2}^{l}
\end{aligned}
$$

The relationship $\{I, H\}_{P}=0$ guarantees that $I$ will be an invariant of the supersymmetric mechanical system. Therefore, conditions making the Poisson bracket vanish (shown in the table 1) must be imposed.

The sum in expression a) in the box 1 ranges over all the permutations of the indices $i, j$ and $k$. We deal with a overdetermined system of partial differential equations: there are 31 PDE relating 15 unknown functions. In the table 1, we have organized the conditions in a step-by-step distribution, i.e. , generically solving conditions in a given box is required to solve the conditions in the following box. In boxes 1 and 2, the equations handled by Hietarinta in the bosonic sector are reproduced. Note too the possibility of the existence of several solutions, giving rise to different supersymmetric invariants. 


\begin{tabular}{|c|c|}
\hline BOX 1 & a) $\sum_{[i j k]} \frac{\partial H^{i j}}{\partial x^{k}}=0$ \\
\hline BOX 2 & a) $H^{i j} \frac{\partial^{2} W}{\partial x^{j} \partial x^{k}} \frac{\partial W}{\partial x^{k}}=\frac{\partial K}{\partial x^{i}}$ \\
\hline BOX 3 & $\begin{array}{l}\text { a) } \epsilon^{j k} \frac{\partial L_{j k}^{i}}{\partial x^{l}}+\epsilon^{j k} \frac{\partial L_{j k}^{l}}{\partial x^{i}}=0 \\
\text { b) } \epsilon^{j k} \frac{\partial M_{j k}^{i}}{\partial x^{l}}+\epsilon^{j k} \frac{\partial M_{j k}^{l}}{\partial x^{i}}=0\end{array}$ \\
\hline BOX 4 & $\begin{array}{l}\text { a) } H^{n j} \frac{\partial^{3} W}{\partial x^{k} \partial x^{l} \partial x^{j}}+i \frac{\partial F_{k l}}{\partial x^{n}}+2 i L_{k m}^{n} \frac{\partial^{2} W}{\partial x^{m} \partial x^{l}}+2 i M_{l j}^{n} \frac{\partial^{2} W}{\partial x^{j} \partial x^{k}}=0 \\
\text { b) } \epsilon^{i j}\left(\frac{\partial^{2} W}{\partial x^{i} \partial x^{k}} F_{k j}-M_{i j}^{k} \frac{\partial^{2} W}{\partial x^{k} \partial x^{l}} \frac{\partial W}{\partial x^{l}}\right)=0 \\
\text { c) } \epsilon^{i j}\left(\frac{\partial^{2} W}{\partial x^{j} \partial x^{k}} F_{i k}+L_{i j}^{l} \frac{\partial^{2} W}{\partial x^{k} \partial x^{l}} \frac{\partial W}{\partial x^{k}}\right)=0\end{array}$ \\
\hline BOX 5 & $\begin{array}{l}\text { a) } \frac{\partial N_{j k}^{i}}{\partial x^{l}}+\frac{\partial N_{j k}^{l}}{\partial x^{i}}=0 \\
\text { b) } \epsilon^{i j} \epsilon^{l k} N_{j k}^{m} \frac{\partial^{3} W}{\partial x^{i} \partial x^{l} \partial x^{m}}=0\end{array}$ \\
\hline BOX 6 & $\begin{array}{l}\text { a) } \epsilon^{i j}\left(\frac{\partial G_{i j}}{\partial x^{l}}-N_{i k}^{l} \frac{\partial^{2} W}{\partial x^{j} \partial x^{k}}\right)=0 \\
\text { b) } \epsilon^{i j}\left(\frac{\partial J_{i j}}{\partial x^{k}}+N_{m j}^{k} \frac{\partial^{2} W}{\partial x^{m} \partial x^{i}}\right)=0 \\
\text { c) } G_{i j} \frac{\partial^{2} W}{\partial x^{j} \partial x^{k}}+J_{k l} \frac{\partial^{2} W}{\partial x^{i} \partial x^{l}}-\frac{1}{2} N_{i k}^{j} \frac{\partial^{2} W}{\partial x^{j} \partial x^{l}} \frac{\partial W}{\partial x^{l}}=0\end{array}$ \\
\hline BOX 7 & a) $\epsilon^{i j} \epsilon^{k l} \frac{\partial S_{i j k l}}{\partial x^{m}}=0$ \\
\hline
\end{tabular}

Table 1: Conditions on I to obtain a supersymmetric invariant. 


\subsection{Second invariant in SuperLiouville models}

In order to solve the above conditions, we proceed in a recurrent way:

i) The equations in BOXES 1 and 2 are sufficient to find $H^{i j}$ and $K$. We recover the information about the second invariant of the purely bosonic sector.

ii) The equations in $B O X 3$ are solved if the independent components of $L_{j k}^{i}$ and $M_{j k}^{i}$ are of the form,

$$
L_{12}^{i}=C \epsilon^{i j} x^{j}+A_{i} \quad M_{12}^{i}=D \epsilon^{i j} x^{j}+B_{i}
$$

where $A_{i}, B_{i}, C$ y $D$ are constants.

iii) The equations in $B O X_{4}$, together with the previous information, lead to the computation of $F_{i j}$. The existence of a solution in equation $4 \mathrm{a}$ ) requires compliance with the identity $\frac{\partial^{2} F_{k l}}{\partial x^{1} \partial x^{2}}=\frac{\partial^{2} F_{k l}}{\partial x^{2} \partial x^{1}}$, which becomes

$$
\epsilon^{m n} \frac{\partial}{\partial x^{m}}\left[L_{j k}^{n} \frac{\partial^{2} W}{\partial x^{j} \partial x^{l}}+M_{j l}^{n} \frac{\partial^{2} W}{\partial x^{k} \partial x^{j}}+\frac{i}{2} H^{n j} \frac{\partial^{3} W}{\partial x^{j} \partial x^{k} \partial x^{l}}\right]=0
$$

Moreover, if we restrict $F_{i j}$ to be symmetric under the exchange of indices and identify $L_{j k}^{i}=M_{j k}^{i}$, equation $4 \mathrm{~b}$ ) becomes equal to $\left.4 \mathrm{c}\right)$. Keeping in mind the formulae $(16),(20),(22)$ and (24), this latter condition and (25) become identities, choosing in each type of Liouville models the appropriate values of the integration constants $A_{i}, B_{i}, C$ and $D$, obtained in the previous point. Thus, the compatibility of the equations is satisfied and we obtain finally $F_{i j}$ from formula a).

$i v$ ) The equations of $B O X E S 5,6$ and 7 are satisfied if we consider:

$$
G_{i j}=J_{i j}=N_{i j k}=S_{i j k l}=0
$$

The second invariant in superLiouville models is of the form

$$
I_{2}=I_{2}^{(B)}+I_{2}^{(F)}
$$

where $I_{2}^{(B)}$ agrees with the purely bosonic second invariant but bearing in mind that the variables have an even Grassmannian character and $I_{2}^{(F)}$ includes terms also involving the fermionic variables $\theta_{\alpha}^{i}$. We find:

\subsubsection{SuperLiouville Models of Type I:}

$$
\begin{aligned}
& I_{2}^{(B)}=\frac{1}{2}\left[\left(x^{2} p_{1}-x^{1} p_{2}\right)^{2}-c^{2} p_{2} p_{2}+\left(x^{2} \frac{\partial W^{(a, b)}}{\partial x^{1}}-x^{1} \frac{\partial W^{(a, b)}}{\partial x^{2}}\right)^{2}-c^{2} \frac{\partial W^{(a, b)}}{\partial x^{2}} \frac{\partial W^{(a, b)}}{\partial x^{2}}\right] \\
& I_{2}^{(F)}=i\left(x^{2} p_{1}-x^{1} p_{2}\right) \theta_{\alpha}^{1} \theta_{\alpha}^{2}+i\left(2 x^{1} \frac{\partial^{2} W^{(a, b)}}{\partial x^{2} \partial x^{2}}-\frac{\partial W^{(a, b)}}{\partial x^{1}}-x^{2} \frac{\partial^{2} W^{(a, b)}}{\partial x^{1} \partial x^{2}}\right) \theta_{1}^{2} \theta_{2}^{2}+
\end{aligned}
$$




$$
\begin{aligned}
& +i\left(-x^{1} x^{2} \frac{\partial^{2} W^{(a, b)}}{\partial x^{2} \partial x^{2}}+x^{2} \frac{\partial W^{(a, b)}}{\partial x^{1}}+x^{2} x^{2} \frac{\partial^{2} W^{(a, b)}}{\partial x^{1} \partial x^{2}}\right)\left(\theta_{1}^{1} \theta_{2}^{2}+\theta_{1}^{2} \theta_{2}^{1}\right)+ \\
& +i\left(-x^{2} \frac{\partial W^{(a, b)}}{\partial x^{2}}-x^{1} x^{2} \frac{\partial^{2} W^{(a, b)}}{\partial x^{1} \partial x^{2}}+x^{2} x^{2} \frac{\partial^{2} W^{(a, b)}}{\partial x^{1} \partial x^{1}}\right) \theta_{1}^{1} \theta_{2}^{1}
\end{aligned}
$$

\subsubsection{SuperLiouville Models of Type II:}

$$
\begin{aligned}
I_{2}^{(B)} & =\frac{1}{2}\left(x^{2} p_{1}-x^{1} p_{2}\right)^{2}+\frac{1}{2}\left(x^{2} \frac{\partial W^{(a, b)}}{\partial x^{1}}-x^{1} \frac{\partial W^{(a, b)}}{\partial x^{2}}\right)^{2} \\
I_{2}^{(F)} & =i\left(x^{2} p_{1}-x^{1} p_{2}\right) \theta_{\alpha}^{1} \theta_{\alpha}^{2}+i x^{2}\left(x^{2} \frac{\partial W^{(a, b)}}{\partial x^{1} \partial x^{1}}-x^{1} \frac{\partial^{2} W^{(a, b)}}{\partial x^{1} \partial x^{2}}-\frac{\partial W^{(a, b)}}{\partial x^{2}}\right) \theta_{1}^{1} \theta_{2}^{1}+ \\
& +i x^{2}\left(x^{2} \frac{\partial^{2} W^{(a, b)}}{\partial x^{1} \partial x^{1}}+\frac{\partial W^{(a, b)}}{\partial x^{1}}-x^{1} \frac{\partial^{2} W^{(a, b)}}{\partial x^{2} \partial x^{2}}\right)\left(\theta_{1}^{1} \theta_{2}^{2}+\theta_{1}^{2} \theta_{2}^{1}\right)+ \\
& +i x^{1}\left(x^{1} \frac{\partial^{2} W^{(a, b)}}{\partial x^{2} \partial x^{2}}-\frac{\partial W^{(a, b)}}{\partial x^{1}}-x^{2} \frac{\partial^{2} W^{(a, b)}}{\partial x^{1} \partial x^{2}}\right) \theta_{1}^{2} \theta_{2}^{2}
\end{aligned}
$$

\subsubsection{SuperLiouville Models of Type III:}

$$
\begin{aligned}
I_{2}^{(B)}= & \left(x^{1} p_{2}-x^{2} p_{1}\right) p_{2}+\left(x^{1} \frac{\partial W^{(a, b)}}{\partial x^{2}}-x^{2} \frac{\partial W^{a, b)}}{\partial x^{1}}\right) \frac{\partial W^{(a, b)}}{\partial x^{2}} \\
I_{2}^{(F)}= & -i p_{2} \theta_{\alpha}^{1} \theta_{\alpha}^{2}-i x^{2} \frac{\partial^{2} W^{(a, b)}}{\partial x^{1} \partial x^{2}} \theta_{1}^{1} \theta_{2}^{1}-i x^{2} \frac{\partial^{2} W^{(a, b)}}{\partial x^{2} \partial x^{2}}\left(\theta_{1}^{1} \theta_{2}^{2}+\theta_{1}^{2} \theta_{2}^{1}\right)+ \\
& +i\left(2 x^{1} \frac{\partial^{2} W^{(a, b)}}{\partial x^{2} \partial x^{2}}-\frac{\partial W^{(a, b)}}{\partial x^{1}}-x^{2} \frac{\partial^{2} W^{(a, b)}}{\partial x^{1} \partial x^{2}}\right) \theta_{1}^{2} \theta_{2}^{2}
\end{aligned}
$$

We observe a common feature in the second invariant of superLiouville models of Type I, II, and III: $l^{12}=x^{1} p_{2}-x^{2} p_{1}$ can be replaced by $j^{12}=x^{1} p_{2}-x^{2} p_{1}+i \theta_{\alpha}^{1} \theta_{\alpha}^{2}$ - invariant only if there is rotational symmetry - and, thus, $s^{12}=i \theta_{\alpha}^{1} \theta_{\alpha}^{2}$ can be interpreted as the spin of the supersymmetric particle. By adding $S_{3}$ to the second invariant in models of Type I and II we obtain a new invariant in the form of:

$$
I_{2}^{\prime}=I_{2}^{(B)}+I_{2}^{(F)}+\frac{1}{4} S_{3}
$$

There is a term,

$$
\frac{1}{2} j^{12} j^{12}=\frac{1}{2}\left(x^{1} p_{2}-x^{2} p_{1}+i \theta_{\alpha}^{1} \theta_{\alpha}^{2}\right)^{2}
$$

in $I_{2}^{\prime}$ with an obvious physical meaning. In models of Type III, $I_{2}$ contains the term $j^{12} p_{2}$ without the need to add anything. 


\subsubsection{SuperLiouville Models of Type IV:}

$$
I_{2}^{(B)}=\frac{1}{2} p_{1} p_{1}+\frac{1}{2} \frac{\partial W^{(a, b)}}{\partial x^{1}} \frac{\partial W^{(a, b)}}{\partial x^{1}} \quad I_{2}^{(F)}=i \frac{\partial^{2} W^{(a, b)}}{\partial x^{1} \partial x^{1}} \theta_{1}^{1} \theta_{2}^{1}
$$

Like $I_{1}^{(F)}, I_{2}^{(F)}$ depends on $b$ : the second invariant in superLiouville models differs for different supersymmetric extensions of the parent Liouville model.

\subsection{Other invariants}

From the pattern shown in the table we also conclude that the generator of R-symmetry $S_{2}$ and their highest non-zero power - $S_{2}^{2}$ in two dimensions - are invariants.

- The condition in BOX 7 is not coupled to the rest of equations in table 1. It sets the only independent component of $S_{i j k l}$ to be constant, $S_{1212}=c$. Thus, as mentioned at the end of sub-Section $\S 2.2$, we check that

$$
S_{3}=-\frac{1}{2} S_{2}^{2}=\theta_{1}^{1} \theta_{1}^{2} \theta_{2}^{1} \theta_{2}^{2}
$$

is a constant of motion, an invariant for all the $\mathcal{N}=2$ supersymmetric mechanical systems with two bosonic degrees of freedom because no restriction on the superpotential has been imposed.

- If all the tensors vanish in the generic expansion of the invariant, except the choice $F_{i j}=\delta_{i j}$, one immediately sees that

$$
S_{2}=i \theta_{1}^{1} \theta_{2}^{1}+i \theta_{1}^{2} \theta_{2}^{2}
$$

is a first integral in two dimensional $\mathcal{N}=2$ supersymmetric mechanical systems.

\section{Supersolutions}

Given the "even", $I_{1}=I_{1}^{(B)}+I_{1}^{(F)}, I_{2}=I_{2}^{(B)}+I_{2}^{(F)}, S_{2}$, and "odd", $Q_{\alpha}$, invariants of a superLiouville mechanical system, the supertrajectories are constrained to satisfy:

$$
\begin{array}{rll}
I_{1}\left(x^{j}, p_{j}, \theta_{\alpha}^{j}\right)=i_{1} & , & I_{2}\left(x^{j}, p_{j}, \theta_{\alpha}^{j}\right)=i_{2} \\
Q_{\alpha}\left(x^{j}, p_{j}, \theta_{\alpha}^{j}\right)=q_{\alpha} & & S_{2}\left(\theta_{\alpha}^{j}\right)=s_{2}
\end{array}
$$

where $i_{1}, i_{2}, q_{\alpha}$, and $s_{2}$ are time-independent arbitrary quantities. The system of equations (31)(32) relates bosonic, $x^{j}(t)$, to fermionic, $\theta_{\alpha}^{j}$, variables; the $x^{j}(t)$ coordinates cannot be ordinary functions; instead, they take values in the even subalgebra $\mathcal{B}_{L}^{e}$ of the underlying Grassmann algebra $\mathcal{B}_{L}$. 


\subsection{The Heumann-Manton method}

To solve the complicated system of equations (31), we propose the method envisaged by Heumann and Manton in [12]. $L=4$ is sufficient for our purposes, so that the identity and the real monomials

$$
\xi_{A} \quad, \quad \xi_{A B}=i \xi_{A} \xi_{B} \quad, \quad \xi_{A B C}=i \xi_{A} \xi_{B} \xi_{C} \quad, \quad \xi_{1234}=-\xi_{1} \xi_{2} \xi_{3} \xi_{4} \quad,
$$

where $A, B, C=1,2,3,4$, provide a basis of $\mathcal{B}_{4}$. The key idea is to expand $x^{j}(t), p_{j}(t)$ and $\theta_{\alpha}^{j}(t)$ on this basis:

$$
\begin{array}{cll}
x^{j}(t)=x_{o}^{j}(t)+x_{A B}^{j}(t) \xi_{A B}+x_{1234}^{j}(t) \xi_{1234} & , & p_{j}(t)=p_{j}^{o}(t)+p_{j}^{A B}(t) \xi_{A B}+p_{j}^{1234}(t) \xi_{1234} \\
\theta_{\alpha}^{j}(t)=\lambda_{\alpha A}^{j}(t) \xi_{A}+\lambda_{\alpha A B C}^{j}(t) \xi_{A B C} & ,
\end{array}
$$

where $x_{o}^{j}(t), p_{j}^{o}(t)$, ( the body ), $x_{A B}^{j}(t), p_{j}^{A B}(t), x_{1234}^{j}(t)$, and $p_{j}^{1234}(t)$ are ordinary function whereas $\lambda_{\alpha A}^{j}(t)$ and $\lambda_{\alpha A B C}^{j}(t)$, are ordinary Majorana spinors. Of course, there is antisymmetry in the $A, B, C$ indices of $x_{A B}^{j}, p_{j}^{A B}$ and $\lambda_{\alpha A B C}^{j}$.

To facilitate the notation, we call $I_{M}^{(B)}=B_{M}\left(x^{j}, p_{j}\right), M=1,2$, and $I_{M}^{(F)}=F_{M}\left(x^{j}, p_{j}, \theta_{\alpha}^{j}\right)$ the terms of the invariants without and with fermionic variables respectively. The expansion of all the invariants

$$
\begin{array}{ccc}
B_{M}\left(x^{j}, p_{j}\right)=B_{M}^{o}+B_{M}^{A B} \xi_{A B}+B_{M}^{1234} \xi_{1234} & , & F_{M}\left(x^{j}, p_{j}\right)=F_{M}^{A B} \xi_{A B}+F_{M}^{1234} \xi_{1234} \\
Q_{\alpha}\left(x^{j}, p_{j}, \theta_{\alpha}^{j}\right)=Q_{\alpha}^{A} \xi_{A}+Q_{\alpha}^{A B C} \xi_{A B C} & , & S_{2}\left(\theta_{\alpha}^{j}\right)=S_{2}^{A B} \xi_{A B}+S_{2}^{1234} \xi_{1234}
\end{array}
$$

together with a parallel expansion of the integration constants,

$$
\begin{array}{rlr}
i_{1}=i_{1}^{o}+i_{1}^{A B} \xi_{A B}+i_{1}^{1234} \xi_{1234} & i_{2}=i_{2}^{o}+i_{2}^{A B} \xi_{A B}+i_{2}^{1234} \xi_{1234} \\
q_{\alpha}=q_{\alpha}^{A} \xi_{A}+q_{\alpha}^{A B C} \xi_{A B C} & , \quad s_{2}=s_{2}^{A B} \xi_{A B}+s_{2}^{1234} \xi_{1234}
\end{array}
$$

permits a layer-by-layer writing of the (31) system:

$$
\begin{array}{rll}
B_{1}^{o}=i_{1}^{o} & , & B_{2}^{o}=i_{2}^{o} \\
B_{1}^{A B}+F_{1}^{A B}=i_{1}^{A B} & , & B_{2}^{A B}+F_{2}^{A B}=i_{2}^{A B} \\
B_{1}^{1234}+F_{1}^{1234}=i_{1}^{1234} & , & B_{2}^{1234}+F_{2}^{1234}=i_{2}^{1234}
\end{array} .
$$

Here, $i_{M}^{o}, i_{M}^{A B}, i_{M}^{1234}, q_{\alpha}^{A}, q_{\alpha}^{A B C}, s_{2}^{A B}$, and $s_{2}^{1234}$ are real numbers and a tedious calculation gives:

$$
\begin{gathered}
B_{M}^{o}=B_{M}\left(x_{o}^{j}, p_{j}^{o}\right) \\
B_{M}^{A B}=\frac{\partial B_{M}}{\partial x^{k}}\left(x_{o}^{j}, p_{j}^{o}\right) x_{A B}^{k}+\frac{\partial B_{M}}{\partial p_{k}}\left(x_{o}^{j}, p_{j}^{o}\right) p_{k}^{A B} \\
B_{M}^{1234}=\frac{\partial B_{M}}{\partial x^{k}}\left(x_{o}^{j}, p_{j}^{o}\right) x_{1234}^{k}+\frac{\partial B_{M}}{\partial p_{k}}\left(x_{o}^{j}, p_{j}^{o}\right) p_{j}^{1234}+\frac{1}{2} \frac{\partial^{2} B_{M}}{\partial x^{k} \partial x^{l}}\left(x_{o}^{j}, p_{j}^{o}\right) \varepsilon_{A B C D} x_{A B}^{k} x_{C D}^{l}+ \\
+\frac{1}{2} \varepsilon_{A B C D}\left(\frac{\partial^{2} B_{M}}{\partial x^{k} \partial p_{l}}\left(x_{o}^{j}, p_{j}^{o}\right) x_{A B}^{k} p_{l}^{C D}+\frac{1}{2} \frac{\partial^{2} B_{M}}{\partial p_{k} \partial x^{l}}\left(x_{o}^{j}, p_{j}^{o}\right) p_{k}^{A B} x_{C D}^{l}+\frac{1}{2} \frac{\partial^{2} B_{M}}{\partial p_{k} \partial p_{l}}\left(x_{o}^{j}, p_{j}^{o}\right) p_{k}^{A B} p_{l}^{C D}\right)
\end{gathered}
$$




$$
\begin{gathered}
F_{1}^{A B}=\frac{\partial^{2} W}{\partial x^{k} \partial x^{l}}\left(x_{o}^{j}\right) \varepsilon_{A B C D} \lambda_{1 C}^{k} \lambda_{2 D}^{l} \\
F_{1}^{1234}=\varepsilon_{A B C D}\left(\frac{\partial^{2} W}{\partial x^{k} \partial x^{l}}\left(x_{o}^{j}\right)\left(\lambda_{1 A}^{k} \lambda_{2 B C D}^{l}+\lambda_{1 A B C}^{k} \lambda_{2 D}^{l}\right)+\frac{\partial^{3} W}{\partial x^{k} \partial x^{l} \partial x^{m}}\left(x_{o}^{j}\right) x_{A B}^{m} \lambda_{1 C}^{k} \lambda_{2 D}^{l}\right) \\
Q_{\alpha}^{A}=p_{k}^{o} \lambda_{\alpha A}^{k}-\varepsilon_{\alpha \beta} \frac{\partial W}{\partial x^{k}}\left(x_{o}^{j}\right) \lambda_{\beta A}^{k} \\
Q_{\alpha}^{A B C}=p_{k}^{o} \lambda_{\alpha A B C}^{k}-\varepsilon_{\alpha \beta} \frac{\partial W}{\partial x^{k}}\left(x_{o}^{j}\right) \lambda_{\beta A B C}^{k}+\varepsilon_{A B C D}\left(p_{k}^{D E} \lambda_{\alpha F}^{k}-\varepsilon_{\alpha \beta} \frac{\partial^{2} W}{\partial x^{k} \partial x^{l}}\left(x_{o}^{k}\right) x_{D E}^{l} \lambda_{\beta F}^{k}\right) \\
S_{2}^{A B}=\varepsilon_{A B C D}\left(\lambda_{1 C}^{1} \lambda_{2 D}^{1}+\lambda_{1 C}^{2} \lambda_{2 D}^{2}\right) \quad, \quad S_{2}^{1234}=\varepsilon_{A B C D}\left(\lambda_{1 A}^{1} \lambda_{2 B C D}^{1}+\lambda_{1 A}^{2} \lambda_{2 B C D}^{2}+\lambda_{1 A B C}^{1} \lambda_{2 D}^{1}+\lambda_{1 A B C}^{2} \lambda_{2 D}^{2}\right)
\end{gathered}
$$

It is not possible to calculate the $F_{2}^{A B}$ and $F_{2}^{1234}$ components of the second invariant in a unified way because they are different for different Types. Nevertheless, the first three Types share a common structure, namely:

$$
F_{2}\left(x^{j}, p_{j}, \theta_{\alpha}^{j}\right)=i l^{12}\left(x_{o}^{j}, p_{j}^{o}\right) \theta_{\alpha}^{1} \theta_{\alpha}^{2}+f^{k l}\left(x_{o}^{j}\right) \theta_{1}^{k} \theta_{2}^{l} \quad,
$$

where $f^{k l}\left(x_{o}^{j}\right)$ can be identified from (27), (28), and (29) in each case. We thus find:

$$
\begin{gathered}
F_{2}^{A B}=\varepsilon_{A B C D}\left(l^{12}\left(x_{o}^{j}, p_{j}^{o}\right) \lambda_{\alpha C}^{1} \lambda_{\alpha D}^{2}-i f^{k l}\left(x_{o}^{j}\right) \lambda_{1 C}^{k} \lambda_{2 D}^{l}\right) \\
F_{2}^{1234}=\varepsilon_{A B C D}\left[l^{12}\left(x_{o}^{j}, p_{j}^{o}\right)\left(\lambda_{\alpha A}^{1} \lambda_{\alpha B C D}^{2}+\lambda_{\alpha A B C}^{1} \lambda_{\alpha D}^{2}\right)+\left(\frac{\partial l^{12}}{\partial x^{k}}\left(p_{j}^{o}\right) x_{A B}^{k}+\frac{\partial l^{12}}{\partial p_{k}}\left(x_{o}^{j}\right) p_{k}^{A B}\right) \lambda_{\alpha C}^{1} \lambda_{\alpha D}^{2}\right]+ \\
+\varepsilon_{A B C D}\left[f^{k l}\left(x_{o}^{j}\right)\left(\lambda_{1 A}^{k} \lambda_{2 B C D}^{l}+\lambda_{1 A B C}^{k} \lambda_{2 D}^{l}\right)-i \frac{\partial f^{k l}}{\partial x^{m}}\left(x_{o}^{j}\right) x_{A B}^{m} \lambda_{1 C}^{k} \lambda_{2 D}^{l}\right]
\end{gathered}
$$

The equations for the basic layer (35) ruling the dynamics of the body of the system can be reduced to quadratures, as in the original Liouville model. Except for Liouville models of Type IV, this not longer holds for equations (36)-(37) in the first and second layers, where the variables describing different degrees of freedom become entangled. Nevertheless, we can be helped by considering equations (32) provided by the fermionic invariants:

$$
\begin{array}{rll}
Q_{\alpha}^{A}=q_{\alpha}^{A} & , & Q_{\alpha}^{A B C}=q_{\alpha}^{A B C} \\
S_{2}^{A B}=s_{2}^{A B} & , & S_{2}^{1234}=s_{2}^{1234}
\end{array}
$$

\subsection{Supersymmetry versus separability}

In Type IV models, the two degrees of freedom completely split in equations (35), (36), (37): we have twice the solutions discussed in Reference [12]. We shall now analyze the situation in the other three cases using the coordinate system where the equations for the basic layer are separable. 


\section{Type I}

Using elliptic coordinates, the invariants of the Type I systems are:

$$
\begin{aligned}
& \xi^{*} B_{1}=\frac{1}{2\left(u^{2}-v^{2}\right)}\left[\left(u^{2}-c^{2}\right)\left(p_{u}^{2}+\left(\frac{d F}{d u}\right)^{2}\right)+\left(c^{2}-v^{2}\right)\left(p_{v}^{2}+\left(\frac{d G}{d v}\right)^{2}\right)\right] \\
& \xi^{*} F_{1}=i\left[\frac{d^{2} F}{d u^{2}}+\frac{u\left(c^{2}-v^{2}\right)}{\left(u^{2}-v^{2}\right)\left(u^{2}-c^{2}\right)} \frac{d F}{d u}-\frac{v\left(c^{2}-v^{2}\right)}{\left(u^{2}-v^{2}\right)\left(u^{2}-c^{2}\right)} \frac{d G}{d v}\right] \vartheta_{1}^{u} \vartheta_{2}^{u} \\
& +i\left[\frac{v}{u^{2}-v^{2}} \frac{d F}{d u}+\frac{u}{u^{2}-v^{2}} \frac{d G}{d v}\right]\left(\vartheta_{1}^{u} \vartheta_{2}^{v}+\vartheta_{1}^{v} \vartheta_{2}^{u}\right) \\
& +i\left[\frac{d^{2} G}{d v^{2}}+\frac{u\left(u^{2}-c^{2}\right)}{\left(u^{2}-v^{2}\right)\left(c^{2}-v^{2}\right)} \frac{d F}{d u}-\frac{v\left(u^{2}-c^{2}\right)}{\left(u^{2}-v^{2}\right)\left(c^{2}-v^{2}\right)} \frac{d G}{d v}\right] \vartheta_{1}^{v} \vartheta_{2}^{v} \\
& \xi^{*} B_{2}=\frac{\left(u^{2}-c^{2}\right)\left(c^{2}-v^{2}\right)}{2\left(u^{2}-v^{2}\right)}\left\{\left[p_{v}^{2}+\left(\frac{d G}{d v}\right)^{2}\right]-\left[p_{u}^{2}+\left(\frac{d F}{d u}\right)^{2}\right]\right\} \\
& \xi^{*} F_{2}=i\left(v p_{u}-u p_{v}\right) \vartheta_{\alpha}^{u} \vartheta_{\alpha}^{v} \\
& -i\left(c^{2}-v^{2}\right)\left[\frac{d^{2} F}{d u^{2}}+\frac{u\left(c^{2}-v^{2}\right)}{\left(u^{2}-v^{2}\right)\left(u^{2}-c^{2}\right)} \frac{d F}{d u}-\frac{v}{u^{2}-c^{2}}\left(\frac{c^{2}-v^{2}}{u^{2}-v^{2}}-1\right) \frac{d G}{d v}\right] \vartheta_{1}^{u} \vartheta_{2}^{u} \\
& +i\left[\frac{u\left(c^{2}-v^{2}\right)}{u^{2}-v^{2}} \frac{d G}{d v}+\frac{v\left(u^{2}-c^{2}\right)}{u^{2}-v^{2}} \frac{d F}{d u}\right]\left(\vartheta_{1}^{u} \vartheta_{2}^{v}+\vartheta_{1}^{v} \vartheta_{2}^{u}\right) \\
& +i\left(u^{2}-c^{2}\right)\left[\frac{d^{2} G}{d v^{2}}+\frac{u}{c^{2}-v^{2}}\left(\frac{u^{2}-c^{2}}{u^{2}-v^{2}}-1\right) \frac{d F}{d u}-\frac{v\left(u^{2}-c^{2}\right)}{\left(u^{2}-v^{2}\right)\left(c^{2}-v^{2}\right)} \frac{d G}{d v}\right] \vartheta_{1}^{v} \vartheta_{2}^{v} \\
& \xi^{*} Q_{\alpha}=p_{u} \vartheta_{\alpha}^{u}-\varepsilon_{\alpha \beta} \frac{d F}{d u} \vartheta_{\beta}^{u}+p_{v} \vartheta_{\alpha}^{v}-\varepsilon_{\alpha \beta} \frac{d G}{d v} \vartheta_{\beta}^{v} \quad, \quad \xi^{*} S_{2}=i\left(u^{2}-v^{2}\right)\left(\frac{\vartheta_{1}^{u} \vartheta_{2}^{u}}{u^{2}-c^{2}}+\frac{\vartheta_{1}^{v} \vartheta_{2}^{v}}{c^{2}-v^{2}}\right)
\end{aligned}
$$

\section{Type III}

In parabolic coordinates the, invariants of the Type III systems read:

$$
\begin{aligned}
\zeta^{*} B_{1} & =\frac{1}{2\left(u^{2}+v^{2}\right)}\left[p_{u}^{2}+\left(\frac{d F}{d u}\right)^{2}+p_{v}^{2}+\left(\frac{d G}{d v}\right)^{2}\right] \\
\zeta^{*} F_{1} & =i\left[\frac{d^{2} F}{d u^{2}}-\frac{u}{u^{2}+v^{2}} \frac{d F}{d u}+\frac{v}{u^{2}+v^{2}} \frac{d G}{d v}\right] \vartheta_{1}^{u} \vartheta_{2}^{u} \\
& -i\left[\frac{u}{u^{2}+v^{2}} \frac{d G}{d v}+\frac{v}{u^{2}+v^{2}} \frac{d F}{d u}\right]\left(\vartheta_{1}^{u} \vartheta_{2}^{v}+\vartheta_{1}^{v} \vartheta_{2}^{u}\right) \\
+ & i\left[\frac{d^{2} G}{d v^{2}}+\frac{u}{u^{2}+v^{2}} \frac{d F}{d u}-\frac{v}{u^{2}+v^{2}} \frac{d G}{d v}\right] \vartheta_{1}^{v} \vartheta_{2}^{v}
\end{aligned}
$$




$$
\begin{aligned}
\zeta^{*} B_{2}=\frac{1}{2\left(u^{2}+v^{2}\right)}\left\{u^{2}\left[p_{v}^{2}+\left(\frac{d G}{d v}\right)^{2}\right]-v^{2}\left[p_{u}^{2}+\left(\frac{d F}{d u}\right)^{2}\right]\right\} \\
\zeta^{*} F_{2}=-i\left(v p_{u}+u p_{v}\right) \vartheta_{\alpha}^{u} \vartheta_{\alpha}^{v}-i v^{2}\left[\frac{d^{2} F}{d u^{2}}-\frac{u}{u^{2}+v^{2}} \frac{d F}{d u}+\left(\frac{v}{u^{2}+v^{2}}-1\right) \frac{d G}{d v}\right] \vartheta_{1}^{u} \vartheta_{2}^{u} \\
-i u v\left[\frac{u}{u^{2}+v^{2}} \frac{d G}{d v}-\frac{v}{u^{2}+v^{2}} \frac{d F}{d u}\right]\left(\vartheta_{1}^{u} \vartheta_{2}^{v}+\vartheta_{1}^{v} \vartheta_{2}^{u}\right) \\
+i u^{2}\left[\frac{d^{2} G}{d v^{2}}+\left(\frac{u}{u^{2}+v^{2}}-1\right) \frac{d F}{d u}-\frac{v}{u^{2}+v^{2}} \frac{d G}{d v}\right] \vartheta_{1}^{v} \vartheta_{2}^{v} \\
\zeta^{*} Q_{\alpha}=p_{u} \vartheta_{\alpha}^{u}-\varepsilon_{\alpha \beta} \frac{d F}{d u} \vartheta_{\beta}^{u}+p_{v} \vartheta_{\alpha}^{v}-\varepsilon_{\alpha \beta} \frac{d G}{d v} \vartheta_{\beta}^{v}, \quad \zeta^{*} S_{2}=i\left(u^{2}+v^{2}\right)\left(\vartheta_{1}^{u} \vartheta_{2}^{u}+\vartheta_{1}^{v} \vartheta_{2}^{v}\right)
\end{aligned}
$$

Use of the expansions

$$
\begin{array}{ccc}
u(t)=u_{o}(t)+u_{A B}(t) \xi_{A B}+u_{1234}(t) \xi_{1234} & , & v(t)=v_{o}(t)+v_{A B}(t) \xi_{A B}+v_{1234}(t) \xi_{1234} \\
p_{u}(t)=p_{u}^{o}(t)+p_{u}^{A B}(t) \xi_{A B}+p_{u}^{1234}(t) \xi_{1234} & , & p_{v}(t)=p_{v}^{o}(t)+p_{v}^{A B}(t) \xi_{A B}+p_{v}^{1234}(t) \xi_{1234} \\
\vartheta_{\alpha}^{u}(t)=\lambda_{\alpha A}^{u}(t) \xi_{A}+\lambda_{\alpha A B C}^{u}(t) \xi_{A B C} & , & \vartheta_{\alpha}^{v}(t)=\lambda_{\alpha A}^{v}(t) \xi_{A}+\lambda_{\alpha A B C}^{v}(t) \xi_{A B C}
\end{array}
$$

in the system of equations ruling the dynamics of the Type I and Type III systems,

$$
\begin{aligned}
& \xi^{*} I_{1}=i_{1} \quad, \quad \xi^{*} I_{2}=i_{2} \quad, \quad \xi^{*} Q_{\alpha}=q_{\alpha} \quad, \quad \xi^{*} S_{2}=s_{2} \\
& \zeta^{*} I_{1}=i_{1} \quad, \quad \zeta^{*} I_{2}=i_{2} \quad, \quad \zeta^{*} Q_{\alpha}=q_{\alpha} \quad, \quad \zeta^{*} S_{2}=s_{2}
\end{aligned}
$$

leads to a layer-by-layer solution of the problem.

In the basic layer, with no Grassman variables at all, the dynamics of the $u$ and $v$ variables are completely independent with respect to each other and a solution by quadratures is at hand. One can easily check that this is not the case in the second and third layer: owing to the Yukawa terms, the dynamics of the $u$ and $v$ variables are no longer independent in the supersymmetric extension of this kind of system.

\section{Type II}

The behaviour of Type II models is identical to the behaviour described above for Type I and III systems. We thus merely list the invariants of this Type of model for completeness using polar coordinates:

$$
\begin{gathered}
\rho^{*} B_{1}=\frac{1}{2}\left[p_{\rho}^{2}+\left(\frac{d F}{d \rho}\right)^{2}\right]+\frac{1}{2 \rho^{2}}\left[p_{\chi}^{2}+\left(\frac{d G}{d \chi}\right)^{2}\right] \\
\rho^{*} F_{1}=i \frac{d^{2} F}{d \rho^{2}} \vartheta_{1}^{\rho} \vartheta_{2}^{\rho}+i\left(\frac{d^{2} G}{d \chi^{2}}+\rho \frac{d F}{d \rho}\right) \vartheta_{1}^{\chi} \vartheta_{2}^{\chi}+\frac{1}{\rho} \frac{d G}{d \chi}\left(\vartheta_{1}^{\rho} \vartheta_{2}^{\chi}+\vartheta_{1}^{\chi} \vartheta_{2}^{\rho}\right)
\end{gathered}
$$




$$
\begin{gathered}
\rho^{*} B_{2}=\frac{1}{2}\left\{p_{\chi}^{2}+\left(\frac{d G}{d \chi}\right)^{2}\right\} \quad, \quad \rho^{*} F_{2}=-i \rho^{2} p_{\chi} \vartheta_{\alpha}^{\rho} \vartheta_{\alpha}^{\chi}+i \rho^{2} \frac{d^{2} G}{d \chi^{2}} \vartheta_{1}^{\chi} \vartheta_{2}^{\chi} \\
\rho^{*} Q_{\alpha}=p_{\rho} \vartheta_{\alpha}^{\rho}-\varepsilon_{\alpha \beta} \frac{d F}{d \rho} \vartheta_{\beta}^{\rho}+p_{\chi} \vartheta_{\alpha}^{\chi}-\varepsilon_{\alpha \beta} \frac{d G}{d \chi} \vartheta_{\beta}^{\chi} \quad, \quad \rho^{*} S_{2}=i\left(\vartheta_{1}^{\rho} \vartheta_{2}^{\rho}+\rho^{2} \vartheta_{1}^{\chi} \vartheta_{2}^{\chi}\right)
\end{gathered}
$$

The expansions

$$
\begin{array}{ccc}
\rho(t)=\rho_{o}(t)+\rho_{A B}(t) \xi_{A B}+\rho_{1234}(t) \xi_{1234} & , & \chi(t)=\chi_{o}(t)+\chi_{A B}(t) \xi_{A B}+\chi_{1234}(t) \xi_{1234} \\
p_{\rho}(t)=p_{\rho}^{o}(t)+p_{\rho}^{A B}(t) \xi_{A B}+p_{\rho}^{1234}(t) \xi_{1234} & , & p_{\chi}(t)=p_{\chi}^{o}(t)+p_{\chi}^{A B}(t) \xi_{A B}+p_{\chi}^{1234}(t) \xi_{1234} \\
\vartheta_{\alpha}^{\rho}(t)=\lambda_{\alpha A}^{\rho}(t) \xi_{A}+\lambda_{\alpha A B C}^{\rho}(t) \xi_{A B C} & , & \vartheta_{\alpha}^{\chi}(t)=\lambda_{\alpha A}^{\chi}(t) \xi_{A}+\lambda_{\alpha A B C}^{\chi}(t) \xi_{A B C}
\end{array}
$$

allow to organize the dynamics in a layer-by-layer structure.

\section{Acknowledgements}

A. Alonso-Izquierdo gratefully acknowledges the support of the spanish Secretaria de Estado de Educacion y Universidades co-financed by the Fondo Social Europeo.

\section{References}

[1] P. Ramond, Phys. Rev. D3, (1971) 2415; A. Neveu and J. Schwarz, Nucl. Phys. B31, (1971) 86.

[2] J. Wess and B. Zumino, Nucl. Phys. B70, (1974) 39.

[3] S. Weinberg, The Quantum Theory of Fields, Volume III, Supersymmetry, Cambridge University Press, (Cambridge) 2000.

[4] E. Witten, Dynamical Aspects of QFT, in Quantum Fields and Strings: a Course for Mathematicians, Volume 2, American Mathematical Society, ( Providence ), 1999.

[5] R. Casalbuoni, Nuovo Cim. 33A (1976) 389-431.

[6] F.A. Berezin, M.S. Marinov, Ann. Phys. 104 (1977) 336.

[7] P.G.O. Freund, Introduction to Supersymmetry, Cambridge University Press (Cambridge) 1986.

[8] B. De Witt, Supermanifolds, Cambridge University Press, (Cambridge) 1992.

[9] G. Junker, Supersymmetric methods in quantum and Statistical Physics, Springer, 1996.

[10] P. Dirac, Lectures on quantum mechanics, Yeshiva Univ. Press, 1964.

[11] G. Junker, S. Matthiesen, J. Phys. A27 (1994) L751. 
[12] R. Heumann, N.S. Manton, Ann. Phys. 284 (2000) 52-88.

[13] N.S. Manton, J. Math. Phys. 40 (1999) 736-750.

[14] R. Heumann, J. Phys. A35 (2002) 7437-7460.

[15] J. Liouville, J. Math. Phys. Appl. 11 (1849), 345. A. Perelomov, Integrable Systems of Classical Mechanics and Lie Algebras, Birkhäuser, (1992).

[16] R. Garnier, Ren. Circ. Mat. Palermo43,(1919)155.

[17] M.S. Plyushchay, hep-th/9808130; Nucl. Phys. B464 (1996) 189-212; Nucl. Phys. B512 (1998) 295-315.

[18] A. Kirchberg, J. Lange, P. Pisani and A. Wipf, Ann. Phys. 303(2003)359-388

[19] A. Andrianov, M. Ioffe and D Nishnianidze, J. Phys. A32(1999) 4641-4654

[20] S. Klishevich and M. Plyushchay, Nucl. Phys. B616 (2001) 403-418

[21] S. Klishevich and M. Plyushchay, Nucl. Phys. B640 (2002) 481-503

[22] J. Hietarinta, Phys. Lett. 93A (1982) 55; Phys. Lett. 96A (1983).

[23] B. Grammaticos, B. Borizzi and B. Padjen, Phys. Lett. 89A (1982) 111.

[24] M.F. Rañada, Phys. Lett. A238 (1998) no. 1, 29-34; J. Math. Phys. 41 (2000) no. 4, 21212134.

[25] A. Alonso-Izquierdo, M.A. González León, J. Mateos Guilarte, Invariants in Supersymmetric Classical Mechanics in Publicaciones de la RSME, Vol.II, pp. 15-28 (2001) and hepth/0004053

[26] J. A. Azcarraga and J. Lukierski, Phys. Lett. B113(1982)170.

[27] A. Alonso-Izquierdo, M.A. González León, J. Mateos Guilarte, J. Phys. A31 (1998) 209.

[28] A. Alonso-Izquierdo, M.A. González León, J. Mateos Guilarte, Phys. Lett. B480 (2000) 373.

[29] A. Alonso-Izquierdo, M.A. González León, J. Mateos Guilarte, Nonlinearity 13 (2000) 1137.

[30] P. Deligne and D. Freed, Supersolutions in Quantum Field Theory: a Course for Mathematicians, Volume 1, American Mathematical Society, ( Providence ), 1999.

[31] M. Tabor, Chaos and integrability in non-linear dynamics, John Wiley, (1989). 